\title{
Competitive Exclusion and Limiting Similarity: A Unified Theory
}

Géza Meszéna (geza.meszena@elte.hu)

Mats Gyllenberg (mats.gyllenberg@helsinki.fi)

Liz Pásztor (lizp@falco.elte.hu)

Johan A. J. Metz (metz@rulsfb.leidenuniv.nl)

\section{Approved by}

Ulf Dieckmann

Program Leader, ADN

August 2005 Institute, its National Member Organizations, or other organizations supporting the work. 


\section{IIASA STUDIES IN ADAPTIVE DYNAMICS No. 101}

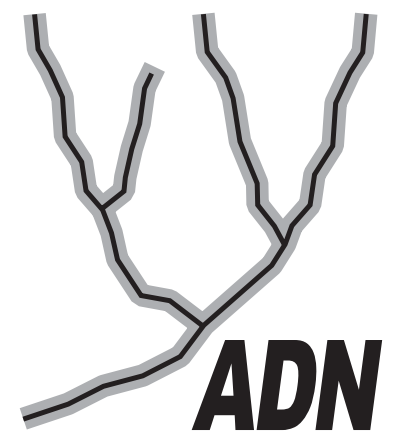

The Adaptive Dynamics Network at IIASA fosters the development of new mathematical and conceptual techniques for understanding the evolution of complex adaptive systems.

Focusing on these long-term implications of adaptive processes in systems of limited growth, the Adaptive Dynamics Network brings together scientists and institutions from around the world with IIASA acting as the central node.

Scientific progress within the network is collected in the IIASA Studies in Adaptive Dynamics series.

No. 1 Metz JAJ, Geritz SAH, Meszéna G, Jacobs FJA, van Heerwaarden JS: Adaptive Dynamics: A Geometrical Study of the Consequences of Nearly Faithful Reproduction. IIASA Working Paper WP-95-099 (1995). van Strien SJ, Verduyn Lunel SM (eds): Stochastic and Spatial Structures of Dynamical Systems, Proceedings of the Royal Dutch Academy of Science (KNAW Verhandelingen), North Holland, Amsterdam, pp. 183-231 (1996).

No. 2 Dieckmann U, Law R: The Dynamical Theory of Coevolution: A Derivation from Stochastic Ecological Processes. IIASA Working Paper WP-96-001 (1996). Journal of Mathematical Biology 34:579-612 (1996).

No. 3 Dieckmann U, Marrow P, Law R: Evolutionary Cycling of Predator-PreyInteractions: Population Dynamics and the Red Queen. IIASA Preprint (1995). Journal of Theoretical Biology 176:91-102 (1995).

No. 4 Marrow P, Dieckmann U, Law R: Evolutionary Dynamics of Predator-Prey Systems: An Ecological Perspective. IIASA Working Paper WP-96-002 (1996). Journal of Mathematical Biology 34:556-578 (1996).

No. 5 Law R, Marrow P, Dieckmann U: On Evolution under Asymmetric Competition. IIASA Working Paper WP-96-003 (1996). Evolutionary Ecology 11:485-501 (1997).

No. 6 Metz JAJ, Mylius SD, Diekmann O: When Does Evolution Optimize? On the Relation Between Types of Density Dependence and Evolutionarily Stable Life History Parameters. IIASA Working Paper WP-96-004 (1996).

No. 7 Ferrière R, Gatto M: Lyapunov Exponents and the Mathematics of Invasion in Oscillatory or Chaotic Populations. Theoretical Population Biology 48:126-171 (1995).

No. 8 Ferrière R, Fox GA: Chaos and Evolution. IIASA Preprint (1996). Trends in Ecology and Evolution 10:480485 (1995)

No. 9 Ferrière R, Michod RE: The Evolution of Cooperation in Spatially Heterogeneous Populations. IIASA Working Paper WP-96-029 (1996). The American Naturalist 147:692717 (1996).

No. 10 van Dooren TJM, Metz JAJ: Delayed Maturation in Temporally Structured Populations with Non-Equilibrium Dynamics. IIASA Working Paper WP-96-070 (1996). Journal of Evolutionary Biology 11:41-62 (1998).
No. 11 Geritz SAH, Metz JAJ, Kisdi É, Meszéna G: The Dynamics of Adaptation and Evolutionary Branching. IIASA Working Paper WP-96-077 (1996). Physical Review Letters 78:2024-2027 (1997).

No. 12 Geritz SAH, Kisdi É, Meszéna G, Metz JAJ: Evolutionary Singular Strategies and the Adaptive Growth and Branching of the Evolutionary Tree. IIASA Working Paper WP-96-114 (1996). Evolutionary Ecology 12:35-57 (1998).

No. 13 Heino M, Metz JAJ, Kaitala V: Evolution of Mixed Maturation Strategies in Semelparous Life-Histories: The Crucial Role of Dimensionality of Feedback Environment. IIASA Working Paper WP-96-126 (1996). Philosophical Transactions of the Royal Society of London Series B 352:1647-1655 (1997).

No. 14 Dieckmann U: Can Adaptive Dynamics Invade? IIASA Working Paper WP-96-152 (1996). Trends in Ecology and Evolution 12:128-131 (1997).

No. 15 Meszéna G, Czibula I, Geritz SAH: Adaptive Dynamics in a 2-Patch Environment: A Simple Model for Allopatric and Parapatric Speciation. IIASA Interim Report IR-97-001 (1997). Journal of Biological Systems 5:265-284 (1997).

No. 16 Heino M, Metz JAJ, Kaitala V: The Enigma of Frequency-Dependent Selection. IIASA Interim Report IR97-061 (1997). Trends in Ecology and Evolution 13:367-370 (1998).

No. 17 Heino M: Management of Evolving Fish Stocks. IIASA Interim Report IR-97-062 (1997). Canadian Journal of Fisheries and Aquatic Sciences 55:1971-1982 (1998).

No. 18 Heino M: Evolution of Mixed Reproductive Strategies in Simple Life-History Models. IIASA Interim Report IR-97063 (1997).

No. 19 Geritz SAH, van der Meijden E, Metz JAJ: Evolutionary Dynamics of Seed Size and Seedling Competitive Ability. IIASA Interim Report IR-97-071 (1997). Theoretical Population Biology 55:324-343 (1999).

No. 20 Galis F, Metz JAJ: Why Are There So Many Cichlid Species? On the Interplay of Speciation and Adaptive Radiation. IIASA Interim Report IR-97-072 (1997). Trends in Ecology and Evolution 13:1-2 (1998). 
No. 21 Boerlijst MC, Nowak MA, Sigmund K: Equal Pay for all Prisoners/ The Logic of Contrition. IIASA Interim Report IR-97-073 (1997). American Mathematical Society Monthly 104:303-307 (1997). Journal of Theoretical Biology 185:281-293 (1997).

No. 22 Law R, Dieckmann U: Symbiosis Without Mutualism and the Merger of Lineages in Evolution. IIASA Interim Report IR-97-074 (1997). Proceedings of the Royal Society of London Series B 265:1245-1253 (1998).

No. 23 Klinkhamer PGL, de Jong TJ, Metz JAJ: Sex and Size in Cosexual Plants. IIASA Interim Report IR-97-078 (1997). Trends in Ecology and Evolution 12:260-265 (1997).

No. 24 Fontana W, Schuster P: Shaping Space: The Possible and the Attainable in RNA Genotype-Phenotype Mapping. IIASA Interim Report IR-98-004 (1998). Journal of Theoretical Biology 194:491-515 (1998).

No. 25 Kisdi É, Geritz SAH: Adaptive Dynamics in Allele Space: Evolution of Genetic Polymorphism by Small Mutations in a Heterogeneous Environment. IIASA Interim Report IR-98-038 (1998). Evolution 53:993-1008 (1999).

No. 26 Fontana W, Schuster P: Continuity in Evolution: On the Nature of Transitions. IIASA Interim Report IR-98-039 (1998). Science 280:1451-1455 (1998).

No. 27 Nowak MA, Sigmund K: Evolution of Indirect Reciprocity by Image Scoring/ The Dynamics of Indirect Reciprocity. IIASA Interim Report IR-98-040 (1998). Nature 393:573-577 (1998). Journal of Theoretical Biology 194:561574 (1998).

No. 28 Kisdi É: Evolutionary Branching Under Asymmetric Competition. IIASA Interim Report IR-98-045 (1998). Journal of Theoretical Biology 197:149-162 (1999).

No. 29 Berger U: Best Response Adaptation for Role Games. IIASA Interim Report IR-98-086 (1998).

No. 30 van Dooren TJM: The Evolutionary Ecology of Dominance-Recessivity. IIASA Interim Report IR-98-096 (1998). Journal of Theoretical Biology 198:519-532 (1999).

No. 31 Dieckmann U, O'Hara B, Weisser W: The Evolutionary Ecology of Dispersal. IIASA Interim Report IR-98-108 (1998). Trends in Ecology and Evolution 14:88-90 (1999).

No. 32 Sigmund K: Complex Adaptive Systems and the Evolution of Reciprocation. IIASA Interim Report IR-98-100 (1998). Ecosystems 1:444-448 (1998).

No. 33 Posch M, Pichler A, Sigmund K: The Efficiency of Adapting Aspiration Levels. IIASA Interim Report IR-98103 (1998). Proceedings of the Royal Society London Series B 266:1427-1435 (1999).

No. 34 Mathias A, Kisdi É: Evolutionary Branching and Coexistence of Germination Strategies. IIASA Interim Report IR-99-014 (1999).

No. 35 Dieckmann U, Doebeli M: On the Origin of Species by Sympatric Speciation. IIASA Interim Report IR-99-013 (1999). Nature 400:354-357 (1999).

No. 36 Metz JAJ, Gyllenberg M: How Should We Define Fitness in Structured Metapopulation Models? Including an Application to the Calculation of Evolutionarily Stable Dispersal Strategies. IIASA Interim Report IR-99-019 (1999). Proceedings of the Royal Society of London Series B 268:499508 (2001)
No. 37 Gyllenberg M, Metz JAJ: On Fitness in Structured Metapopulations. IIASA Interim Report IR-99-037 (1999). Journal of Mathematical Biology 43:545-560 (2001).

No. 38 Meszéna G, Metz JAJ: Species Diversity and Population Regulation: The Importance of Environmental Feedback Dimensionality. IIASA Interim Report IR-99-045 (1999).

No. 39 Kisdi É, Geritz SAH: Evolutionary Branching and Sympatric Speciation in Diploid Populations. IIASA Interim Report IR-99-048 (1999).

No. 40 Ylikarjula J, Heino M, Dieckmann U: Ecology and Adaptation of Stunted Growth in Fish. IIASA Interim Report IR-99-050 (1999). Evolutionary Ecology 13:433-453 (1999).

No. 41 Nowak MA, Sigmund K: Games on Grids. IIASA Interim Report IR-99-038 (1999). Dieckmann U, Law R, Metz JAJ (eds): The Geometry of Ecological Interactions: Simplifying Spatial Complexity, Cambridge University Press, Cambridge, UK, pp. 135-150 (2000).

No. 42 Ferrière R, Michod RE: Wave Patterns in Spatial Games and the Evolution of Cooperation. IIASA Interim Report IR-99-041 (1999). Dieckmann U, Law R, Metz JAJ (eds): The Geometry of Ecological Interactions: Simplifying Spatial Complexity, Cambridge University Press, Cambridge, UK, pp. 318-332 (2000).

No. 43 Kisdi É, Jacobs FJA, Geritz SAH: Red Queen Evolution by Cycles of Evolutionary Branching and Extinction. IIASA Interim Report IR-00-030 (2000). Selection 2:161$176(2001)$.

No. 44 Meszéna G, Kisdi É, Dieckmann U, Geritz SAH, Metz JAJ: Evolutionary Optimisation Models and Matrix Games in the Unified Perspective of Adaptive Dynamics. IIASA Interim Report IR-00-039 (2000). Selection 2:193-210 (2001).

No. 45 Parvinen K, Dieckmann U, Gyllenberg M, Metz JAJ: Evolution of Dispersal in Metapopulations with Local Density Dependence and Demographic Stochasticity. IIASA Interim Report IR-00-035 (2000). Journal of Evolutionary Biology 16:143-153 (2003).

No. 46 Doebeli M, Dieckmann U: Evolutionary Branching and Sympatric Speciation Caused by Different Types of Ecological Interactions. IIASA Interim Report IR-00-040 (2000). The American Naturalist 156:S77-S101 (2000).

No. 47 Heino M, Hanski I: Evolution of Migration Rate in a Spatially Realistic Metapopulation Model. IIASA Interim Report IR-00-044 (2000). The American Naturalist 157:495$511(2001)$.

No. 48 Gyllenberg M, Parvinen K, Dieckmann U: Evolutionary Suicide and Evolution of Dispersal in Structured Metapopulations. IIASA Interim Report IR-00-056 (2000). Journal of Mathematical Biology 45:79-105 (2002).

No. 49 van Dooren TJM: The Evolutionary Dynamics of Direct Phenotypic Overdominance: Emergence Possible, Loss Probable. IIASA Interim Report IR-00-048 (2000). Evolution 54: 1899-1914 (2000).

No. 50 Nowak MA, Page KM, Sigmund K: Fairness Versus Reason in the Ultimatum Game. IIASA Interim Report IR00-57 (2000). Science 289:1773-1775 (2000).

No. 51 de Feo O, Ferrière R: Bifurcation Analysis of Population Invasion: On-Off Intermittency and Basin Riddling. IIASA Interim Report IR-00-074 (2000). International Journal of Bifurcation and Chaos 10:443-452 (2000). 
No. 52 Heino M, Laaka-Lindberg S: Clonal Dynamics and Evolution of Dormancy in the Leafy Hepatic Lophozia Silvicola. IIASA Interim Report IR-01-018 (2001). Oikos 94:525-532 (2001).

No. 53 Sigmund K, Hauert C, Nowak MA: Reward and Punishment in Minigames. IIASA Interim Report IR-01-031 (2001). Proceedings of the National Academy of Sciences of the USA 98:10757-10762 (2001).

No. 54 Hauert C, De Monte S, Sigmund K, Hofbauer J: Oscillations in Optional Public Good Games. IIASA Interim Report IR-01-036 (2001).

No. 55 Ferrière R, Le Galliard J: Invasion Fitness and Adaptive Dynamics in Spatial Population Models. IIASA Interim Report IR-01-043 (2001). Clobert J, Dhondt A, Danchin E, Nichols J (eds): Dispersal, Oxford University Press, pp. 57-79 (2001).

No. 56 de Mazancourt C, Loreau M, Dieckmann U: Can the Evolution of Plant Defense Lead to Plant-Herbivore Mutualism. IIASA Interim Report IR-01-053 (2001). The American Naturalist 158: 109-123 (2001).

No. 57 Claessen D, Dieckmann U: Ontogenetic Niche Shifts and Evolutionary Branching in Size-Structured Populations. IIASA Interim Report IR-01-056 (2001). Evolutionary Ecology Research 4:189-217 (2002).

No. 58 Brandt H: Correlation Analysis of Fitness Landscapes. IIASA Interim Report IR-01-058 (2001).

No. 59 Dieckmann U: Adaptive Dynamics of Pathogen-Host Interacations. IIASA Interim Report IR-02-007 (2002). Dieckmann U, Metz JAJ, Sabelis MW, Sigmund K (eds): Adaptive Dynamics of Infectious Diseases: In Pursuit of Virulence Management, Cambridge University Press, Cambridge, UK, pp. 39-59 (2002).

No. 60 Nowak MA, Sigmund K: Super- and Coinfection: The Two Extremes. IIASA Interim Report IR-02-008 (2002). Dieckmann U, Metz JAJ, Sabelis MW, Sigmund K (eds): Adaptive Dynamics of Infectious Diseases: In Pursuit of Virulence Management, Cambridge University Press, Cambridge, UK, pp. 124-137 (2002).

No. 61 Sabelis MW, Metz JAJ: Perspectives for Virulence Management: Relating Theory to Experiment. IIASA Interim Report IR-02-009 (2002). Dieckmann U, Metz JAJ, Sabelis MW, Sigmund K (eds): Adaptive Dynamics of Infectious Diseases: In Pursuit of Virulence Management, Cambridge University Press, Cambridge, UK, pp. 379-398 (2002).

No. 62 Cheptou P, Dieckmann U: The Evolution of SelfFertilization in Density-Regulated Populations . IIASA Interim Report IR-02-024 (2002). Proceedings of the Royal Society of London Series B 269:1177-1186(2002).

No. 63 Bürger R: Additive Genetic Variation Under Intraspecific Competition and Stabilizing Selection: A Two-Locus Study. IIASA Interim Report IR-02-013 (2002). Theoretical Population Biology 61:197-213 (2002).

No. 64 Hauert C, De Monte S, Hofbauer J, Sigmund K: Volunteering as Red Queen Mechanism for Co-operation in Public Goods Games. IIASA Interim Report IR-02-041 (2002). Science 296:1129-1132 (2002).

No. 65 Dercole F, Ferrière R, Rinaldi S: Ecological Bistability and Evolutionary Reversals under Asymmetrical Competition. IIASA Interim Report IR-02-053 (2002). Evolution 56:1081-1090 (2002).
No. 66 Dercole F, Rinaldi S: Evolution of Cannibalistic Traits: Scenarios Derived from Adaptive Dynamics. IIASA Interim Report IR-02-054 (2002). Theoretical Population Biology 62:365-374 (2002).

No. 67 Bürger R, Gimelfarb A: Fluctuating Environments and the Role of Mutation in Maintaining Quantitative Genetic Variation. IIASA Interim Report IR-02-058 (2002). Genetical Research 80:31-46 (2002).

No. 68 Bürger R: On a Genetic Model of Intraspecific Competition and Stabilizing Selection. IIASA Interim Report IR02-062 (2002). Amer. Natur. 160:661-682 (2002).

No. 69 Doebeli M, Dieckmann U: Speciation Along Environmental Gradients. IIASA Interim Report IR-02-079 (2002). Nature 421:259-264 (2003).

No. 70 Dercole F, Irisson J, Rinaldi S: Bifurcation Analysis of a Prey-Predator Coevolution Model. IIASA Interim Report IR-02-078 (2002). SIAM Journal on Applied Mathematics 63:1378-1391 (2003).

No. 71 Le Galliard J, Ferrière R, Dieckmann U: The Adaptive Dynamics of Altruism in Spatially Heterogeneous Populations. IIASA Interim Report IR-03-006 (2003). Evolution 57:1-17 (2003).

No. 72 Taborsky B, Dieckmann U, Heino M: Unexpected Discontinuities in Life-History Evolution under SizeDependent Mortality. IIASA Interim Report IR-03-004 (2003). Proceedings of the Royal Society of London Series B 270:713-721 (2003).

No. 73 Gardmark A, Dieckmann U, Lundberg P: LifeHistory Evolution in Harvested Populations: The Role of Natural Predation. IIASA Interim Report IR-03-008 (2003). Evolutionary Ecology Research 5:239-257 (2003).

No. 74 Mizera F, Meszéna G: Spatial Niche Packing, Character Displacement and Adaptive Speciation Along an Environmental Gradient. IIASA Interim Report IR-03-062 (2003). Evolutionary Ecology Research 5: 363-382 (2003).

No. 75 Dercole F: Remarks on Branching-Extinction Evolutionary Cycles. IIASA Interim Report IR-03-075 (2003). Journal of Mathematical Biology 47: 569-580 (2003).

No. 76 Hofbauer J, Sigmund K: Evolutionary Game Dynamics. IIASA Interim Report IR-03-078 (2003). Bulletin of the American Mathematical Society 40: 479-519 (2003).

No. 77 Ernande B, Dieckmann U, Heino M: Adaptive Changes in Harvested Populations: Plasticity and Evolution of Age and Size at Maturation. IIASA Interim Report IR03-058 (2003). Proceedings of the Royal Society of London Series B-Biological Sciences, 271: 415-423 (2004).

No. 78 Hanski I, Heino M: Metapopulation-Level Adaptation of Insect Host Plant Preference and Extinction-Colonization Dynamics in Heterogeneous Landscapes. IIASA Interim Report IR-03-028 (2003). Theoretical Population Biology 63:309-338 (2003).

No. 79 van Doorn G, Dieckmann U, Weissing FJ: Sympatric Speciation by Sexual Selection: A Critical Re-Evaluation. IIASA Interim Report IR-04-003 (2004). American Naturalist 163: 709-725 (2004).

No. 80 Egas M, Dieckmann U, Sabelis MW: Evolution Restricts the Coexistence of Specialists and Generalists - the Role of Trade-off Structure. IIASA Interim Report IR-04-004 (2004). American Naturalist, 163: 518-531 (2004). 
No. 81 Ernande B, Dieckmann U: The Evolution of Phenotypic Plasticity in Spatially Structured Environments: Implications of Intraspecific Competition, Plasticity Costs, and Environmental Characteristics. IIASA Interim Report IR-04-006 (2004). Journal of Evolutionary Biology 17 (3): 613-628 (2004).

No. 82 Cressman R, Hofbauer J: Measure Dynamics on a One-Dimensional Continuous Trait Space: Theoretical Foundations for Adaptive Dynamics. IIASA Interim Report IR04-016 (2004).

No. 83 Cressman R: Dynamic Stability of the Replicator Equation with Continuous Strategy Space. IIASA Interim Report IR-04-017 (2004).

No. 84 Ravigné V, Olivieri I, Dieckmann U: Implications of Habitat Choice for Protected Polymorphisms. IIASA Interim Report IR-04-005 (2004). Evolutionary Ecology Research 6: 125-145 (2004).

No. 85 Nowak MA, Sigmund K: Evolutionary Dynamics of Biological Games. IIASA Interim Report IR-04-013 (2004). Science 303: 793-799 (2004).

No. 86 Vukics A, Asbóth J, Meszéna G: Speciation in Multidimensional Evolutionary Space. IIASA Interim Report IR-04-028 (2004). Physical Review, 68: 041-903 (2003).

No. 87 de Mazancourt C, Dieckmann U: Trade-off Geometries and Frequency-dependent Selection. IIASA Interim Report IR-04-039 (2004). American Naturalist, 164: 765-778 (2004).

No. 88 Cadet CR, Metz JAJ, Klinkhamer PGL: Size and the Not-So-Single Sex: disentangling the effects of size on sex allocation. IIASA Interim Report IR-04-084 (2004). American Naturalist, 164: 779-792 (2004).

No. 89 Rueffler C, van Dooren TJM, Metz JAJ: Adaptive Walks on Changing Landscapes: Levins' Approach Extended. IIASA Interim Report IR-04-083 (2004). Theoretical Population Biology, 65: 165-178 (2004).

No. 90 de Mazancourt C, Loreau M, Dieckmann U: Understanding Mutualism When There is Adaptation to the Partner. IIASA Interim Report IR-05-016 (2005). Journal of Ecology, 93: 305-314 (2005).
No. 91 Dieckmann U, Doebeli M: Pluralism in Evolutionary Theory. IIASA Interim Report IR-05-017 (2005).

No. 92 Doebeli M, Dieckmann U, Metz JAJ, Tautz D: What We Have Also Learned. IIASA Interim Report IR-05-018 (2005). Evolution, 59: 691-695 (2005).

No. 93 Egas M, Sabelis MW, Dieckmann U: Evolution of Specialization and Ecological Character Displacement of Herbivores Along a Gradient of Plant Quality. IIASA Interim Report IR-05-019 (2005). Evolution, 59: 507-520 (2005).

No. 94 Le Galliard J, Ferrière R, Dieckmann U: Adaptive Evolution of Social Traits: Origin, Trajectories, and Correlations of Altruism and Mobility. IIASA Interim Report IR05-020 (2005). American Naturalist, 165: 206-224 (2005).

No. 95 Doebeli M, Dieckmann U: Adaptive Dynamics as a Mathematical Tool for Studying the Ecology of Speciation Processes. IIASA Interim Report IR-05-022 (2005).

No. 96 Brandt H, Sigmund K: The Logic of Reprobation: Assessment and Action Rules for Indirect Reciprocity. IIASA Interim Report IR-04-085 (2004). Journal of Theoretical Biology 231: 475-486 (2004).

No. 97 Hauert C, Haiden N, Sigmund K: The Dynamics of Public Goods. IIASA Interim Report IR-04-086 (2004). Discrete and Continuous Dynamical Systems - Series B, 4:575587 (2004).

No. 98 Meszéna G, Gyllenberg M, Jacobs FJA, Metz JAJ: Dynamics of Similar Populations: The Link between Population Dynamics and Evolution. IIASA Interim Report IR-05026 (2005).

No. 99 Meszéna G: Adaptive Dynamics: The Continuity Argument. IIASA Interim Report IR-05-032 (2005).

No. 100 Brännström NA, Dieckmann U: Evolutionary Dynamics of Altruism and Cheating Among Social Amoebas. IIASA Interim Report IR-05-039 (2005). Proceedings of the Royal Society London Series B, 272: 1609-1616 (2005).

No. 101 Meszéna G, Gyllenberg M, Pasztor L, Metz JAJ: Competitive Exclusion and Limiting Similarity: A Unified Theory. IIASA Interim Report IR-05-040 (2005).

Issues of the IIASA Studies in Adaptive Dynamics series can be obtained at www.iiasa.ac.at/Research/ADN/Series.html or by writing to adn@iiasa.ac.at. 


\section{Contents}

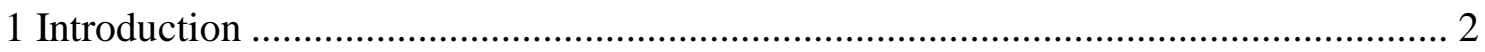

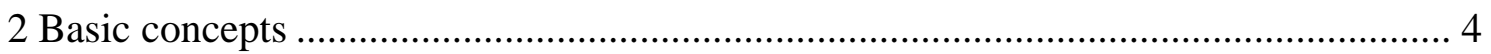

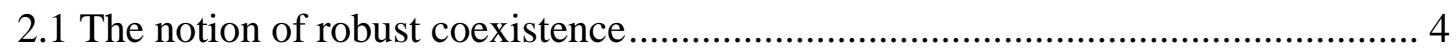

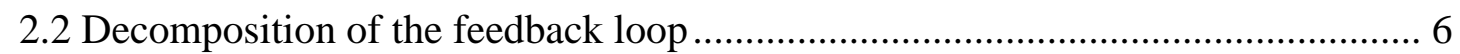

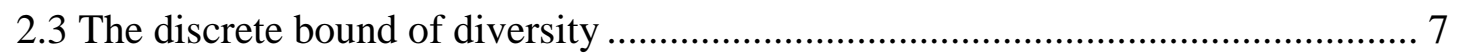

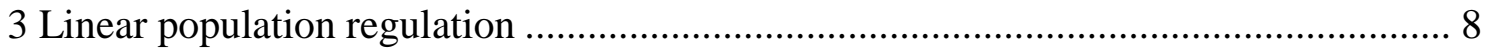

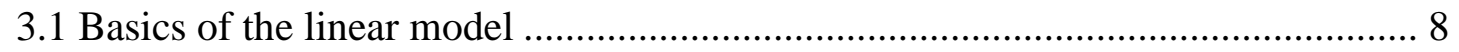

3.2 Strength of population regulation versus robustness of coexistence .................... 10

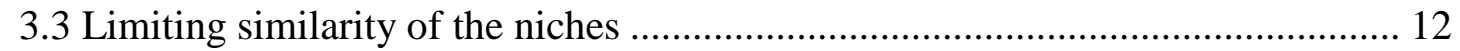

3.4 Niche overlap in case of a resource continuum .............................................. 15

3.5 Structural instability of Roughgarden's example .............................................. 17

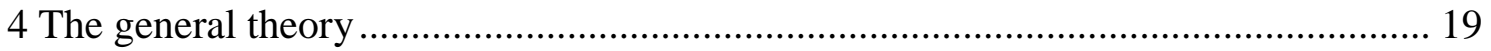

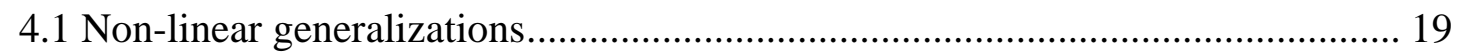

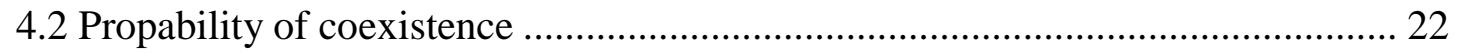

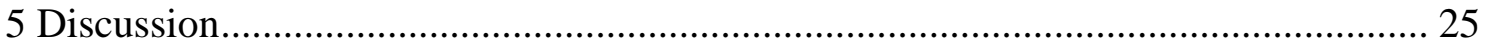

5.1 Competitive exclusion and limiting similarity ................................................... 25

5.2 Connection to the dynamics of adaptation ......................................................... 26

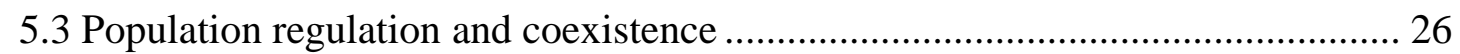

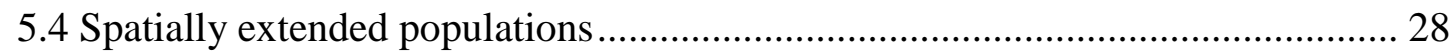

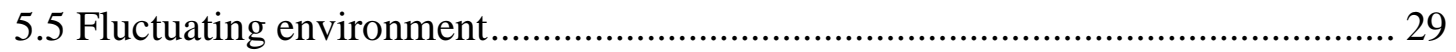

5.6 Niche space as a map for competitive exclusion ................................................. 30

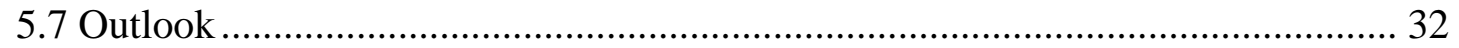

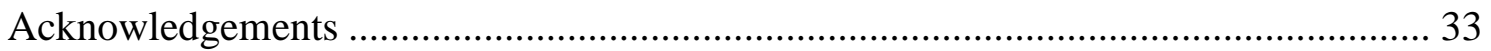

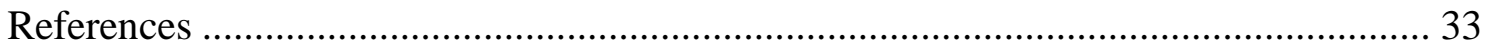

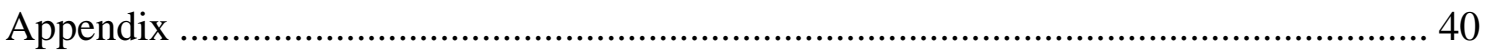




\title{
Competitive exclusion and limiting similarity: a unified theory
}

\author{
Theoretical Population Biology, accepted for publication
}

\author{
Géza Meszéna \\ Department of Biological Physics, Eötvös University, Pázmány Péter sétány 1A, \\ H-1117 Budapest, Hungary \\ Mats Gyllenberg \\ Rolf Nevanlinna Institute, Department of Mathematics and Statistics, \\ University of Helsinki, FIN-00014 Helsinki, Finland \\ Liz Pásztor \\ Department Genetics, Eötvös University, Pázmány Péter sétány 1C, H-1117 \\ Budapest, Hungary
}

Johan A. J. Metz

Institute of Biology, Leiden University, van der Klaauw Laboratory, P.O. Box 9516, NL-2300 RA Leiden, the Netherlands Adaptive Dynamics Network, International Institute for Applied Systems Analysis, A-2361 Laxenburg, Austria

\begin{abstract}
Robustness of coexistence against changes of parameters is investigated in a modelindependent manner through analyzing the feed-back loop of population regulation. We define coexistence as a fixed point of the community dynamics with no population having zero size. It is demonstrated that the parameter range allowing coexistence shrinks and disappears when the Jacobian of the dynamics decreases to zero. A general notion of regulating factors/variables is introduced. For each population, its impact and sensitivity niches are defined as the differential impact on, and the differential sensitivity towards, the regulating variables, respectively. Either similarity of the impact niches, or similarity of the sensitivity niches, result in a small Jacobian and in a reduced likelihood of coexistence. For the case of a resource continuum, this result reduces to the usual "limited niche overlap" picture for both kinds of niche. As an extension of these ideas to the coexistence of infinitely many species, we demonstrate that Roughgarden's example for coexistence of a continuum of populations is structurally unstable.
\end{abstract}


Key words:

population regulation, competitive exclusion, coexistence, limiting similarity, niche

\section{Introduction}

We expect struggle for life between the kinds attempting to occupy the same niche and peaceful coexistence between the species established in different niches (Gause, 1934). This principle of "competitive exclusion" has become a cornerstone of ecological thinking while at the same time it has remained highly controversial. The basic models were introduced by MacArthur and Levins in the middle of the sixties both for discrete and for continuous resources. These models differ considerably in mathematical setup and sophistication.

The discrete models (MacArthur and Levins, 1964; Tilman, 1982) consider competition for a finite number of distinct resources and state that the number of coexisting species should not exceed the number of resources they compete for. This conclusion has a sound mathematical foundation: To have structurally stable solution, i.e., a solution that does not disappear on the slightest change of the model specification, the number of equations describing the population dynamical equilibrium should not exceed the number of unknowns.

With some risk of becoming tautological, we can relax the assumption of resource competition by counting all the factors behaving like resources (Levin, 1970; Armstrong \& McGehee, 1980, Heino et al., 1997). Limited practical usefulness is the price for theoretical robustness. It is a rare biological situation where the resources, or the regulating factors, are easy to count and low in number. In most cases, very many environmental factors that are potentially regulating are present. Which are the really important ones? Which of them should be considered as distinct from the others (cf. Abrams, 1988)? On the other hand, if only the limiting resources are counted, their number often turns out to be too low to explain species diversity in a constant environment (Hutchinson, 1959).

The classical continuous model (MacArthur and Levins, 1967) studies the partitioning of a continuous scale of resources, e.g. seeds of different sizes. In this case, strictly speaking, an infinite number of different resources are present, i.e., each seed size has to be considered as a different resource. Consequently,

Email addresses: geza.meszena@elte.hu (Géza Meszéna), mats.gyllenberg@helsinki.fi (Mats Gyllenberg), lizp@falco.elte.hu (Liz Pásztor), metz@rulsfb.leidenuniv.nl (Johan A. J. Metz). 
the argument used for the discrete situation cannot be applied to bound the number of species in the continuous case. Still, we do not expect an infinite number of species to coexist. The classical concept of "limiting similarity" (Hutchinson, 1959), based on the study of the Lotka-Volterra competition model (MacArthur and Levins, 1967), states that the resource scale is partitioned between the species. The width of the "resource utilization function" of a species is expected to set the width of a single partition, referred to as the "niche breadth". The allowed similarity of the coexisting species is limited and the number of them is bounded by the number of possible partitions. It seems to be reasonable to consider one partition of the resource scale as a single resource, distinct from the rest. Unfortunately, as yet no mathematical theory has appeared that fully captures this intuition in a general way: Again, the question is, to what extent the resources should differ to be counted as different? Neither has any general conclusion emerged when the later studies went beyond the original Lotka-Volterra framework. These studies actually resulted in a fading away of the hope of finding a model-independent lower bound to similarity (Abrams, 1983).

The status of the limiting similarity principle is unclear even for the original Lotka-Volterra model. May (1973, p. 158) found that the limit of similarity of two coexisting species can be arbitrarily small if their respective carrying capacities are chosen sufficiently similar. Yodzis (1989, p. 125) states that, contrary to the two species case, there is a strict lower bound to similarity for three species. Probably, the most drastic blow against limiting similarity occurred when Roughgarden (1979, p. 534-536) provided an example of coexistence of a continuum of types in the Lotka-Volterra model. While the example was intended to describe the phenotype distribution within a single species, it can be interpreted in the context of species coexistence. An infinite number of different resources does allow coexistence of an infinite number of species. The example thus seems to violate the whole idea of limiting similarity (Maynard Smith \& Szathmáry, 1995).

Even though limiting similarity and resource partitioning failed to earn the status of a mathematical theory, they have remained widely accepted concepts in ecology (Begon et al., 1996, p. 300). However, if limiting similarity were just an artifact of some specific mathematical models, we would not be allowed to use it as a basis of biological reasoning. Without limiting similarity, the practical relevance of competitive exclusion would be constrained to the simplest cases of population regulation (Rosenzweig, 1995, p. 127). If we could not safely assume competitive exclusion between the variants of the same species, even the Darwinian concept of natural selection would lose its basis. The goal of the present paper is to make the mathematical step from the solid ground of competitive exclusion in the discrete case to establish the general existence of limiting similarity in a well-defined sense. 
The key issue of species coexistence is the necessity for mechanisms stabilizing it (Chesson, 2000b). This vantage point allow us to investigate the problem independent of specific model assumptions. We start from May's observation (May, 1973, 1974) that the more similar two species are, the more narrow is the range of $K_{1} / K_{2}$ permitting coexistence (May, $1973 ; K_{1}$ and $K_{2}$ stand for the two carrying capacities. See also a similar analysis by Vandermeer, 1975, which uses the intrinsic growth rates, instead of the carrying capacities as a reference.) We generalize this statement beyond its original framework of a Lotka-Volterra type model and to an arbitrary number of species. Limiting similarity is interpreted as a shrinking likelihood of coexistence with increasing similarity. Especially, we demonstrate that the Roughgarden type of continuous coexistence, as it is called, generally becomes impossible on the slightest change of the model. To relate limiting similarity to resource usage, or to the regulating factors, and to the concept of niche, we will apply Leibold's (1995) distinction between the two legs of the population-environment interaction. We restrict our mathematical analysis to fixed point attractors in a constant environment.

The theory is presented in several steps. After introducing our central notions in Section 2, we demonstrate the basic issues of limiting similarity and establish the connection between the discrete and the continuous cases via a linear model in Section 3. Then, in Section 4, the non-linear, model-independent, generalization is presented. Finally, we discuss the wider context of our work, in Section 5. Background mathematics is summarized for the convenience of the interested reader in the Appendix.

\section{Basic concepts}

\subsection{The notion of robust coexistence}

Populations coexist when the long term growth rates of all of them are simultaneously zero, i.e., the births just compensate for the deaths in each of them. Coexistence of any given set of populations can be achieved in many parameter-rich models by adjusting the parameters. The problem of coexistence becomes non-trivial only when one rejects parameter fine-tuning and requires the population dynamical equilibrium point to exist for a considerable range of the parameters. In a given environment, the wider this range is, the more likely the coexistence is. We will refer to this property as robustness of coexistence. Robustness of coexistence is measured by the volume of the set of parameter values permitting coexistence. In special, but important, situations this volume shrinks to zero and robustness is lost completely. Then, coexistence can be achieved only for special choices of the parameters. We will 
refer to such coexistence as structurally unstable. It has probability zero to occur in the real word.

As we will see, robust coexistence is a consequence of feedbacks (or stabilizing mechanisms, Chesson, 2000b), to be called population regulation, with the potential of adjusting the birth and death rates (Figure 1). The growth rates are nullified at different values of the external environmental parameters by different combinations of the population sizes. When the population regulation is weak, changing the population sizes within the acceptable (i.e., positive) range can compensate only for small changes of the external parameters. Therefore the robustness of a particular pattern of coexistence is weak, or lost, when the underlying population regulation is weak, or lost.

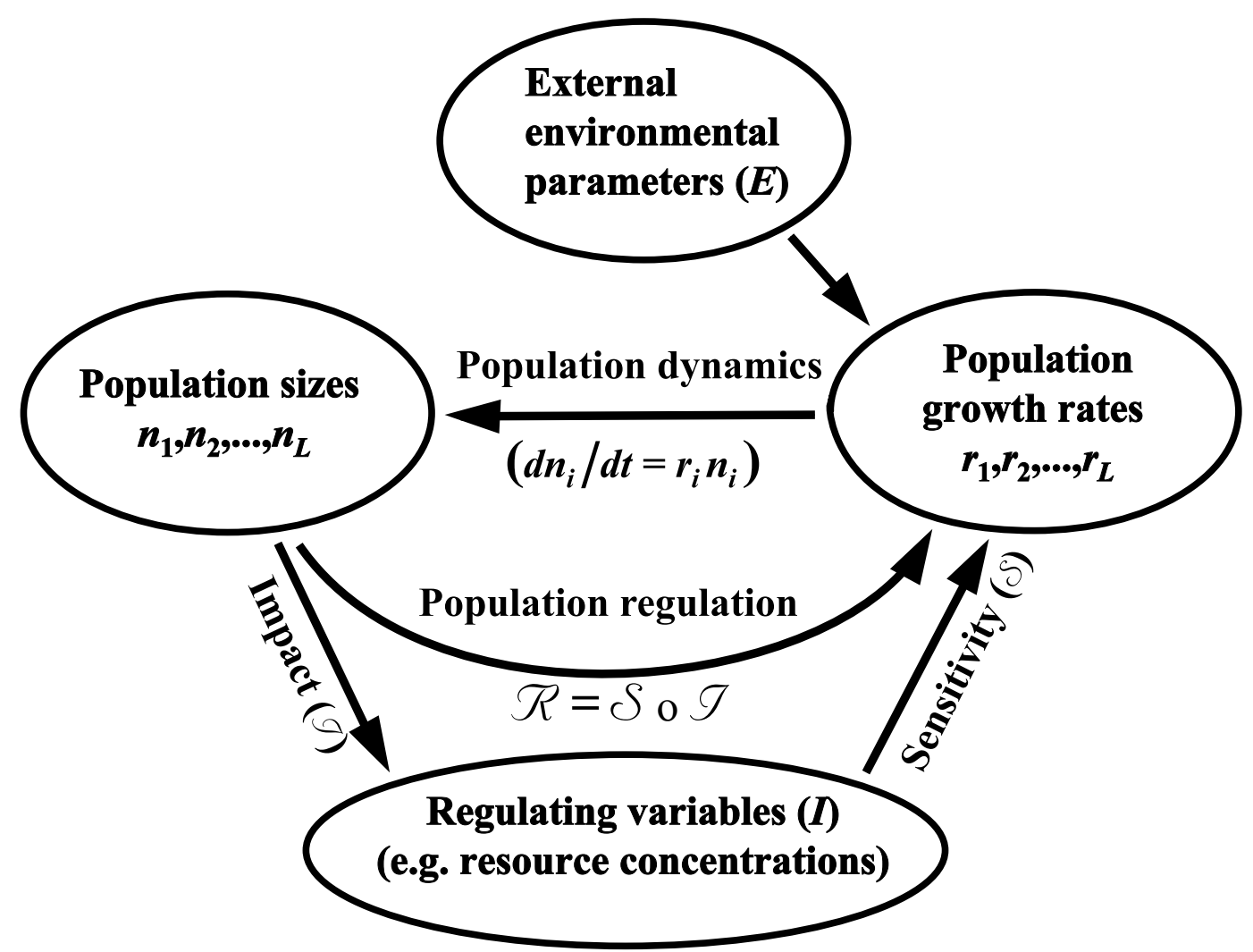

Fig. 1. The general scheme of an environmental feedback loop. The dynamics of the populations are determined by the growth rates. They, in turn, are determined by the population sizes via population regulation $\mathcal{R}$. Population regulation is mediated by the regulating variables collected into the vector $\boldsymbol{I}$. The map $\mathcal{R}$ is a composition of the population impact $\mathcal{I}$ on the regulating variables and the population sensitivity $\mathcal{S}$ towards these variables. The external environmental parameters, collected in the vector $\boldsymbol{E}$, affect also the growth rates. However, these parameters are not involved in the feedback loop as they are independent of the population sizes. The elements of $\boldsymbol{E}$ and $\boldsymbol{I}$, together, are often referred to as limiting factors. 


\subsection{Decomposition of the feedback loop}

While resource limitation is the archetypical mechanism of population regulation, many other types of interactions, like predation, infection, etc., can be at the root of the unavoidable regulation. For a general treatment, it is useful to introduce the notion of regulating variables (or regulating factors, Case, 2000, p. 146; Krebs 2001, p. 288). The regulating variables, collected into the vector $\boldsymbol{I}$, are the variables mediating the feedback. Each element of the vector $\boldsymbol{I}$ depends on the population sizes and affects some of the demographic parameters of the individuals in the populations. Regulating factors are also referred to as "environmental interaction variables", or just "environmental variables" (Heino et al., 1997; Diekmann et al., 2001, 2003; Vukics et al., 2003).

Regulating variables should be clearly distinguished from the external environmental parameters (like temperature, humidity, stress, etc.), which are collected into the vector $\boldsymbol{E}$. Elements of $\boldsymbol{E}$ may affect the populations but are not affected by the populations, so they are not involved in the feedback loop. Turchin (2003, p. 398) uses the terms endogeneous and exogeneous factors for the elements of $\boldsymbol{I}$ and $\boldsymbol{E}$, respectively. The distinction between resources and conditions (Begon et al., 1996) is analogous, but see the Discussion.

As a matter of definition, we suppose that the vectors $\boldsymbol{E}$ and $\boldsymbol{I}$ together represent a complete description of the environment affecting the demographic parameters of the individuals. Specifically, all the interactions between the individuals are mediated through $\boldsymbol{I}$. Elements of the vectors $\boldsymbol{I}$ and $\boldsymbol{E}$ together are often referred to as limiting factors, change of which can cause the extinction of the population (Case, 2000, p. 146.; Krebs, 2001). Note that a limiting resource is always regulating.

In the mathematical treatment we restrict our attention to unstructured populations and describe the state of the community by the population sizes $n_{1}, n_{2}, \ldots, n_{L}$ of the $L$ coexisting species. (See Section 5.4 for the discussion of the spatial structure of the community.) These sizes determine the values of the regulating variables:

$$
\mathcal{I}: \quad\left(n_{1}, n_{2}, \ldots, n_{L}\right) \mapsto \boldsymbol{I}=\mathcal{I}\left(n_{1}, n_{2}, \ldots, n_{L}\right) .
$$

The map $\mathcal{I}$ will be referred to as the impact function (or output map in the terminology of Diekmann et al. 2001, 2003). In turn, the regulating variables, at a given $\boldsymbol{E}$, determine the population growth rates $r_{i}$ :

$$
\mathcal{S}: \quad \boldsymbol{I} \mapsto\left(r_{1}(\boldsymbol{E}, \boldsymbol{I}), r_{2}(\boldsymbol{E}, \boldsymbol{I}), \ldots, r_{L}(\boldsymbol{E}, \boldsymbol{I})\right)
$$

We will refer to the dependence relation $\mathcal{S}$ as the sensitivity function.

Composition of the impact and the sensitivity maps determines the population 
regulation $\mathcal{R}$ which is the dependence of the growth rates on the population sizes at fixed $\boldsymbol{E}$ :

$$
\mathcal{R}=\mathcal{S} \circ \mathcal{I}: \quad\left(n_{1}, n_{2}, \ldots, n_{L}\right) \mapsto\left(r_{1}(\boldsymbol{E}, \boldsymbol{I}), r_{2}(\boldsymbol{E}, \boldsymbol{I}), \ldots, r_{L}(\boldsymbol{E}, \boldsymbol{I})\right)
$$

The population regulation map determines the population dynamics according to the equations

$$
\frac{1}{n_{i}} \cdot \frac{d n_{i}}{d t}=r_{i}\left(\boldsymbol{E}, \mathcal{I}\left(n_{1}, n_{2}, \ldots, n_{L}\right)\right) \quad(i=1,2, \ldots, L) .
$$

An easy calculation leads to the equation

$$
\frac{d\left(\frac{n_{i}}{n_{j}}\right)}{d t}=\left(r_{i}-r_{j}\right) \frac{n_{i}}{n_{j}} \quad(i, j=1,2, \ldots, L)
$$

showing that the ratio of the sizes of the $i$ th and the $j$ th populations is governed by the difference between their growth rates.

In a thought experiment, and sometimes in a real one, one can fix the regulating variables. Doing so, the feedback loop opens up, population regulation ceases to operate and the individuals reproduce and die independently. In this case the equations of population dynamics (4) becomes linear with fixed values of the growth rates $r_{i}$. Then, the populations grow exponentially and the ratio of population sizes changes monotonically according to the differences in the growth rates [Eq. (5)]. For instance, if resource limitation creates the only interaction between the individuals, continuous replenishing of the resources leads to unlimited exponential growth of the now independent populations.

\subsection{The discrete bound of diversity}

The set of equilibrium equations

$$
r_{i}(\boldsymbol{E}, \boldsymbol{I})=0 \quad(i=1,2, \ldots, L)
$$

consists of $L$ equations for $\operatorname{dim} \boldsymbol{I}$ unknowns. Generically, they can be solved only if $L \leq \operatorname{dim} \boldsymbol{I}$. A solution may exist for $L>\operatorname{dim} \boldsymbol{I}$, but such a solution is structurally unstable. If the smallest set of regulating variables is chosen,

$$
D=\operatorname{dim} \boldsymbol{I}
$$

can be regarded as the dimension of population regulation (Meszéna \& Metz, 1999; Heino et al., 1997, Diekmann et al., 2003). Using this quantity, one can state the following. 
Discrete competitive exclusion principle

Structurally stable coexistence of $L$ populations requires

$$
L \leq D
$$

where $D$ is the dimension of population regulation.

A bound of this type is often referred to as the competitive exclusion principle. Without stressing the difference between the limiting and the regulating factors, its generality was recognized already by Levin (1970). (See also Armstrong \& McGehee, 1980; Heino et al., 1997; Meszéna \& Metz, 1999.) We use the qualifier discrete to distinguish from the more general meaning of the same term (c.f. Hardin, 1960).

In the case of pure resource competition, i.e., when resource shortage is the only source of interaction between individuals, the quantities of the resources are the regulating variables and $D$ is the number of different resources. As the elements of $\boldsymbol{E}$ are parameters, rather than unknowns, of the equilibrium equations, they do not count in this context. If a change in the environment does not influence the feedback loop it does not affect the maximal number of coexisting species, either.

As a corollary, no robust coexistence is possible for $D=1$ (Metz et al., 1996b). If all populations are limited by the same resource, the species with the lowest equilibrium resource concentration on its own out-competes all others $\left(R^{*}\right.$ rule, Tilman, 1982, p. 43). If all populations affect the resource equally, the sum of the population sizes can be regarded as the sole regulating variable (density-dependent, or $K$, selection, MacArthur, 1962).

As the infinite number of different resources in a resource continuum demonstrates, the dimension of regulation is not necessarily finite. The discrete version of competitive exclusion is useless in this case.

\section{Linear population regulation}

\subsection{Basics of the linear model}

In this section we investigate the coexistence of $L$ species in the case of linear regulation. As an arbitrary choice, let the origin $\boldsymbol{I}=\mathbf{0}$ of the space of regulating variables correspond to the absence of the populations of our interest.

Assume that the population sizes affect $\boldsymbol{I}$ linearly: 


$$
\boldsymbol{I}=n_{1} \boldsymbol{C}_{1}+n_{2} \boldsymbol{C}_{2}+\cdots+n_{L} \boldsymbol{C}_{L}
$$

Here, the $D$ dimensional vectors $\boldsymbol{C}_{1}, \boldsymbol{C}_{2} \ldots, \boldsymbol{C}_{L}$ denote the per capita environmental impact of the corresponding populations. In case of pure resource competition, the components of $\boldsymbol{I}$ measure the depletion of the different resources caused by the presence of the populations.

Moreover, we suppose also that the growth rates depend linearly on the regulating variables, e.g. on the resource depletions:

$$
r_{i}=r_{0 i}(\boldsymbol{E})-\boldsymbol{S}_{i} \cdot \boldsymbol{I} \quad(i=1,2, \ldots, L),
$$

where the "." denotes scalar product. $r_{0 i}(\boldsymbol{E})$ is the innate capacity of increase, which depends only on the external parameters $\boldsymbol{E}$. The $D$ dimensional vectors $\boldsymbol{S}_{1}, \boldsymbol{S}_{2} \ldots, \boldsymbol{S}_{L}$ describe the sensitivity of the populations to the regulating variables. The minus sign was introduced to harmonize with the depletion interpretation of $\boldsymbol{I}$ for resource competition.

The two vectors related to the $i$ th population, $\boldsymbol{C}_{i}$ and $\boldsymbol{S}_{i}$, characterize the interaction of this population with the regulating factors. These vectors will be referred to as impact and sensitivity niche vectors, respectively. They are related to, but not identical to, Leibold's (1995) concept of impact and requirement niches. See the Discussion for the connection to Huchinson's (1978) notion of niche.

For finite dimensional regulation, the $k$ th component $C_{i k}$ of the impact niche vector $\boldsymbol{C}_{i}$ measures the per capita impact of the $i$ th population on the $k$ th regulating variable $I_{k}$. Similarly, $S_{i k}$ measures the sensitivity of this population towards $I_{k}$. We will examine the infinite dimensional case in Section 3.4.

Equations (9) and (10) together lead to a Lotka-Volterra type population regulation:

$$
r_{i}\left(\boldsymbol{E}, \mathcal{I}\left(n_{1}, n_{2}, \ldots, n_{L}\right)\right)=r_{0 i}(\boldsymbol{E})-\sum_{j=1}^{L} a_{i j} n_{j}
$$

for each $i$, where

$$
a_{i j}=\boldsymbol{S}_{i} \cdot \boldsymbol{C}_{j} \quad(i, j=1,2, \ldots, L)
$$

are the elements of the community matrix $\boldsymbol{a}$. Positive elements of $\boldsymbol{a}$ represent competitive interactions. The competition coefficients used in the conventional Lotka-Volterra formulation are $a_{i j} / a_{i i}$ (see e.g. Vandermeer, 1975).

The set of equilibrium equations (6) can be solved uniquely, and in a struc- 
turally stable way, if the Jacobian determinant

$$
J=\operatorname{det} \boldsymbol{a}
$$

of the system is different from zero. Then, the solution is

$$
n_{i}=\sum_{j=1}^{L} a_{i j}^{-1} r_{0 j}=\frac{1}{J} \sum_{j=1}^{L} \operatorname{adj}(a)_{i j} r_{0 j} \quad(i=1,2, \ldots, L),
$$

where $a_{i j}^{-1}$ and $\operatorname{adj}(a)_{i j}$ are the elements of the inverse and of the adjoint matrix of $\boldsymbol{a}$ (Anton, 1984, p. 80), respectively. (The adjoint matrix is not to be confused with the complex conjugate of the transposed matrix, which is usually also called the adjoint matrix.) The solution is biologically meaningful, i.e., corresponds to coexistence of populations only if all $n_{i}$ are positive.

\subsection{Strength of population regulation versus robustness of coexistence}

Here we show that the robustness of coexistence (i.e., the volume of the permitting set of parameters) decreases zero gradually when $J \rightarrow 0$. Let

$$
U=\left\{\left(n_{1}, n_{2}, \ldots, n_{L}\right) \mid 0<n_{i}<n_{\max } \text { for } i=1,2, \ldots, L\right\}
$$

be the set of admissible community states, i.e., the set of potential combinations of positive population sizes bellow the maximal $n_{\max }$, which can be arbitrarily high. Then,

$$
\left(r_{01}, r_{02}, \ldots, r_{0 L}\right) \in \boldsymbol{a} U
$$

specifies the combinations of $r_{0 i}$ values allowing coexistence, where $\boldsymbol{a} U$ represents the set of possible values of $\boldsymbol{a n}$ when $\boldsymbol{n} \in U$. Using $\mathcal{V}$ to denote volume,

$$
\mathcal{V}(\boldsymbol{a} U)=|J| \mathcal{V}(U)
$$

(Apostol, 1962, pp. 84-86; Case, 2000, pp. 420-422, Edwards, Jr., 1973, p. 245) which proves our claim. Figure 2 illustrates the two-dimensional case, where $\boldsymbol{a} U$ is a parallelogram. ( $n_{\max }$ is not represented.)

Equation (17) is a simple consequence of the geometrical interpretation of a determinant: The linear operation defined by the matrix $\boldsymbol{a}$ maps the unit cube into a parallelepiped of volume $|J|$. This volume serves as a measure of the strength of population regulation on the community level. It is large if the parallelepiped is wide in each direction, i.e., when each population size affects at least some growth rates differently enough and each growth rate is affected differently enough by at least some population sizes.

Obviously, whenever the population regulation is weak, the inverse dependence (related to the matrix $\boldsymbol{a}^{-1}$ ) will be strong. If a large change of the population 

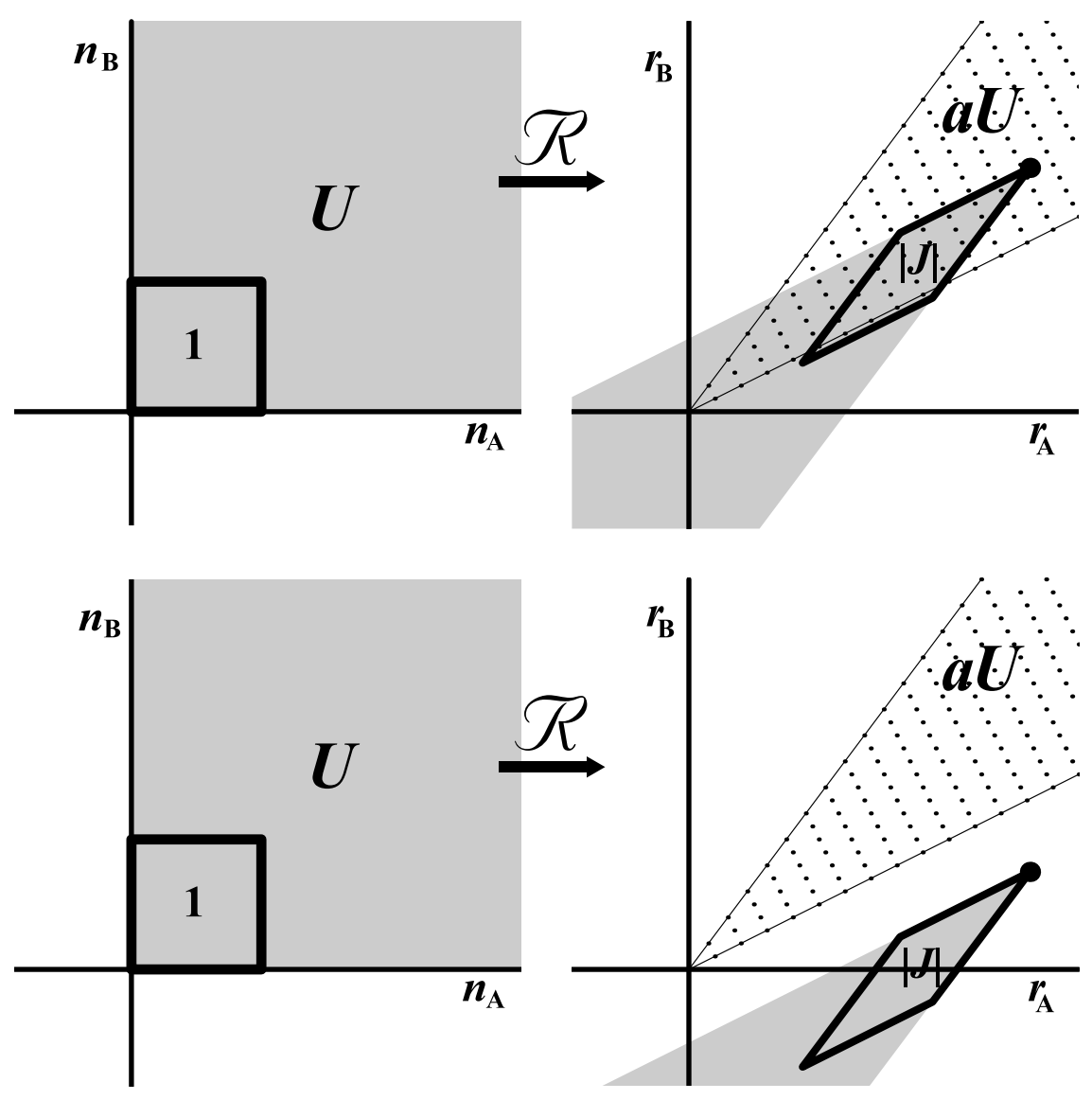

Fig. 2. Linear model for two populations. Top row: coexistence is possible. Bottom row: coexistence is impossible. Left panes: space of population sizes $\boldsymbol{n}=\left(n_{\mathrm{A}}, n_{\mathrm{B}}\right)$, the gray area corresponds to the biologically relevant region $U$, where the population sizes are positive (cf. Eq. 15, the maximal population size $n_{\max }$ is not represented here). Right panes: space of growth rates $\boldsymbol{r}=\left(r_{\mathrm{A}}, r_{\mathrm{B}}\right)$. The population regulation $\mathcal{R}$ maps from the $\boldsymbol{n}$ space to the $\boldsymbol{r}$ space. The gray area of the right plane is the image of the gray area of the left pane, i.e., the set of the values of growth rates, which are reachable by varying the population sizes. The unit square of the $\boldsymbol{n}$ space is mapped to a parallelogram of area $|J|$ in the $\boldsymbol{r}$ space. The population dynamical equilibrium corresponds to the origin of the $\boldsymbol{r}$ space. Coexistence is possible, if the origin is in the gray region. This happens when the intrinsic growth rates $\boldsymbol{r}^{0}=\left(r_{1}^{0}, r_{2}^{0}\right)$, denoted by the large dot, are in the dotted region representing $\boldsymbol{a} U$, where $\boldsymbol{a}$ is the population matrix (cf. Eq. 16). Coexistence is unlikely when the dotted area is small, i.e., when $|J|$ is small.

sizes leads to a small change of the growth rates, then the equilibrium population sizes will be very sensitive to the factors affecting the growth rates. This sensitivity is represented in Equation (14) by the denominator $J$. The extreme sensitivity of the population sizes, when $|J|$ is small, implies that a small change of the parameters could drive some of the populations into extinction. That is, weak population regulation leads to non-robust coexistence. 


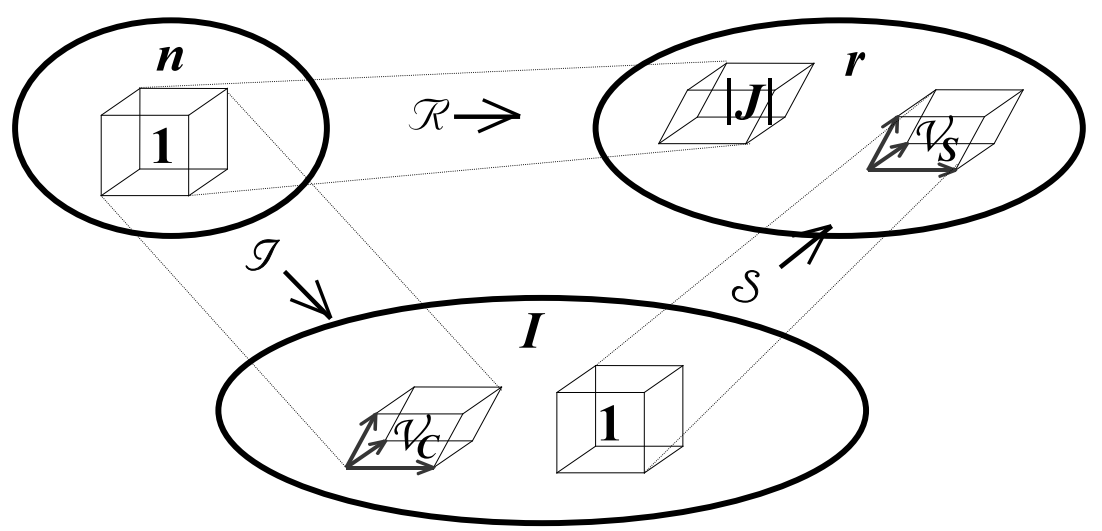

Fig. 3. The volume measures of the population regulation. The environmental impact function $\mathcal{I}$ maps the unit cube into a parallelepiped of volume $\mathcal{V}_{\boldsymbol{C}}$ spanned by the impact niche vectors. The sensitivity function $\mathcal{S}$ maps the unit cube into another parallelepiped, spanned by the sensitivity niche vectors, the volume of which is $\mathcal{V}_{\boldsymbol{S}}$. The combination of these two maps, the population regulation $\mathcal{R}$, maps the unit cube into a parallelepiped of volume $|J|$.

In the limiting case of $J=0$, robustness is lost entirely. The equilibrium equations are solvable only for special choices of $r_{0 i}$. Coexistence, if exists, is structurally unstable for $J=0$.

\subsection{Limiting similarity of the niches}

We want to explore the loss of robustness, discussed in the previous paragraph, in terms of the niche vectors. Obviously, population regulation becomes weak if either the environmental impact or the environmental sensitivity becomes weak.

The strength of the population regulation $\mathcal{R}$ was measured by the volume $|J|$ of the image of the unit cube under $\mathcal{R}$. We need an analogous measure for the impact map $\mathcal{I}$ and for the sensitivity map $\mathcal{S}$. The impact function $\mathcal{I}$ maps the unit cube into the $L$ dimensional parallelepiped spanned by the impact niche vectors $\boldsymbol{C}_{i}$ while the sensitivity function $\mathcal{S}$ maps the unit cube into a parallelepiped spanned by the sensitivity niche vectors $\boldsymbol{S}_{i}$ (Figure 3 ). The volume of these parallelepipeds will be denoted by $\mathcal{V}_{\boldsymbol{C}}$ and $\mathcal{V}_{\boldsymbol{S}}$, respectively.

Note that the volumes $\mathcal{V}_{\boldsymbol{C}}$ and $\mathcal{V}_{\boldsymbol{S}}$ are not determinants, because the maps $\mathcal{I}$ and $\mathcal{S}$ operate between spaces of different dimensionality. The related mathematics is summarized in the Appendix. In the main text, we rely on the clear intuitive notion of the volume in a self-contained way.

The following statement establishes the expected connection between the strenght of impact and sensitivity on the one hand and the strenght of the 
regulation on the other hand.

\section{Proposition: Regulation strength}

$$
|J| \leq \mathcal{V}_{S} \cdot \mathcal{V}_{C}
$$

i.e., either weak impact or weak sensitivity leads to diminished population regulation.

Proof of Proposition. Let $\hat{\boldsymbol{C}}$ is the $D \times L$ matrix composed by the column vectors $\boldsymbol{C}_{i}$ and $\hat{\boldsymbol{S}}$ is the $L \times D$ matrix composed by the row vectors $\boldsymbol{S}_{j}$;

$$
a=\hat{S} \cdot \hat{C}
$$

For $L=D$, they are square matrices and

$$
|J|=|\operatorname{det} \hat{\boldsymbol{S}}| \cdot|\operatorname{det} \hat{\boldsymbol{C}}|=\mathcal{V}_{\boldsymbol{S}} \cdot \mathcal{V}_{\boldsymbol{C}}
$$

For $D>L$, observe that only the $D$-dimensional linear subspace, containing the $\boldsymbol{S}_{i}$ vectors, is relevant. We use a prime to denote the projection onto this subspace. Obviously, $\mathcal{V}_{\boldsymbol{S}^{\prime}}=\mathcal{V}_{\boldsymbol{S}}$ and $\mathcal{V}_{\boldsymbol{C}^{\prime}} \leq \mathcal{V}_{\boldsymbol{C}}$. Then,

$$
|J|=\mathcal{V}_{S^{\prime}} \cdot \mathcal{V}_{C^{\prime}} \leq \mathcal{V}_{S} \cdot \mathcal{V}_{\boldsymbol{C}}
$$

Finally, note that $|J|=\mathcal{V}_{\boldsymbol{C}}=\mathcal{V}_{\boldsymbol{S}}=0$ for $D<L$.

The volumes $\mathcal{V}_{\boldsymbol{C}}$ and $\mathcal{V}_{\boldsymbol{S}}$ measure the dissimilarity of the impact and the sensitivity niche vectors, respectively, in the linear sense. If the vectors $\boldsymbol{C}_{i}$ $(i=1,2, \ldots, L)$ are linearly dependent then $\mathcal{V}_{\boldsymbol{C}}=0$. A small value of $\mathcal{V}_{\boldsymbol{C}}$ represents a situation in which the vectors are close to being linearly dependent. Specifically, similarity of any two or more of the impact niche vectors leads to small $\mathcal{V}_{\boldsymbol{C}}$. Analogously, similarity between the sensitivity niche vectors results in a small value of $\mathcal{V}_{\boldsymbol{S}}$ and linear dependence of them results in $\mathcal{V}_{\boldsymbol{S}}=0$. According to the Proposition, as robust coexistence requires a large enough value of $|J|$, it requires also large enough values for $\mathcal{V}_{\boldsymbol{S}}$ and $\mathcal{V}_{\boldsymbol{C}}$. It is not realistic to suppose that an extreme smallness of $\mathcal{V}_{\boldsymbol{C}}$, i.e., the similarity of the impact vectors, is compensated by an extremely large $\mathcal{V}_{\boldsymbol{S}}$, or vice versa.

Expressed differently, all the impact niche vectors and all the sensitivity niche vectors should be different enough, otherwise population regulation is weak and coexistence is restricted to a narrow range of the parameter values. In more qualitative terms, populations have to differ both in their effects on the regulating variables and in their dependence on these variables (cf. Abrams, 1988).

For $L=2$, the volume $\mathcal{V}_{\boldsymbol{S}}$ reduces to the area of the parallelogram spanned 

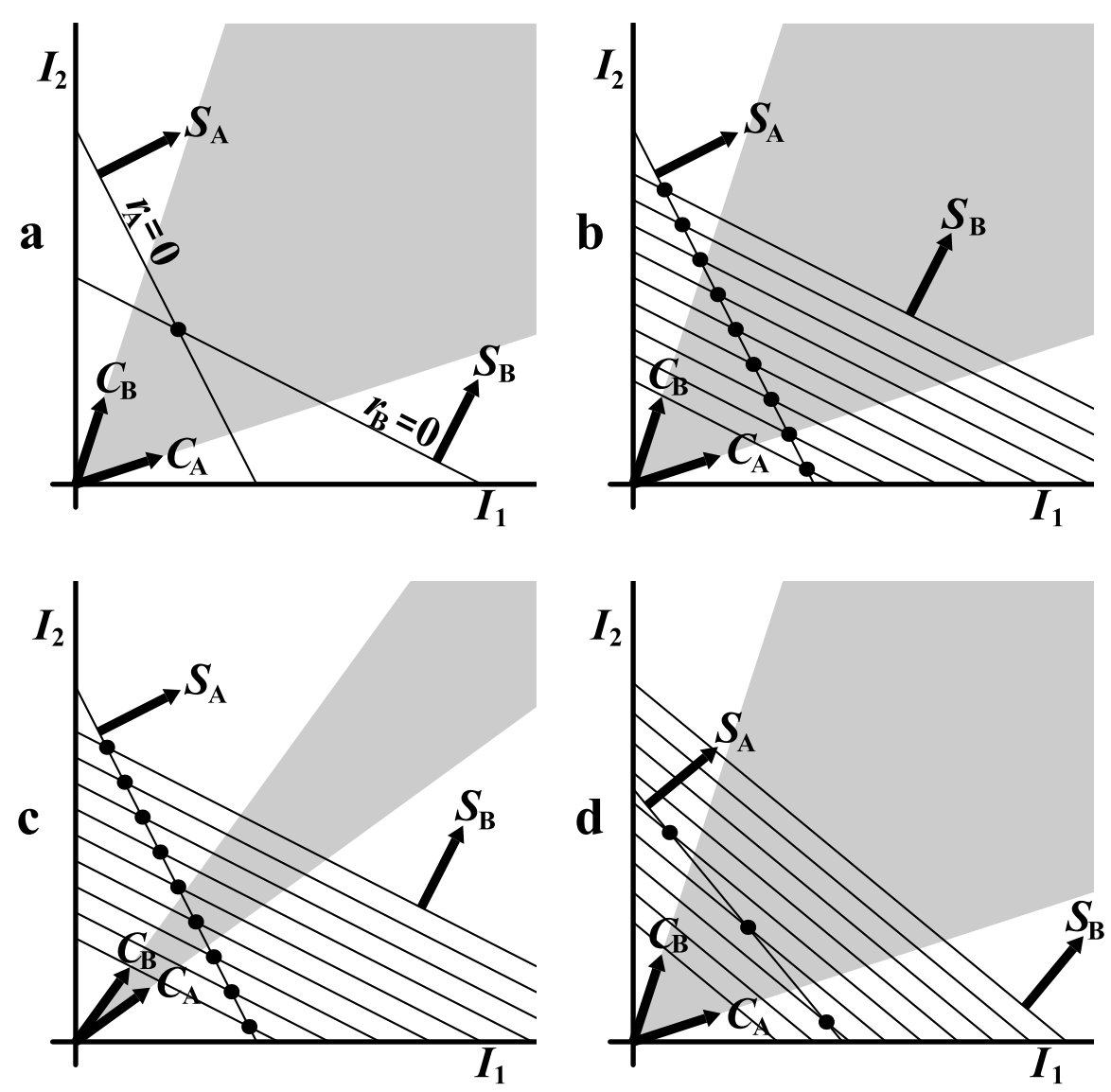

Fig. 4. (a) Equilibrium of two species, A and B, with two dimensional linear population regulation. The axes represent the regulating variables, the origin is the ecological vacuum. The impact niche vectors $\boldsymbol{C}_{\mathrm{A}}$ and $\boldsymbol{C}_{\mathrm{B}}$ determine the change of the regulating variables as a consequence of adding a single individual to the corresponding population. Non-negative population sizes result in the values of the regulating variables within the wedge between these vectors (gray). The two solid lines are the Zero Net Growth Isocline-s (ZNGI, Tilman, 1982) corresponding to the two equilibrium equations, $r_{\mathrm{A}}=0$ and $r_{\mathrm{B}}=0$, of the two species. They are perpendicular to the sensitivity niche vectors $\boldsymbol{S}_{\mathrm{A}}$ and $\boldsymbol{S}_{\mathrm{B}}$. The intersection point of the ZNGIs (large dot) must lie within the gray region to describe biologically realistic coexistence. (b) The equilibrium for several different values of $r_{0 \mathrm{~B}}$. Some of them lie outside the allowed region and correspond to negative population sizes. (c) When the impact niche vectors are very similar, the allowed region is very narrow and most of the equilibrium points lie outside of it. (d) Here the sensitivity niche vectors are the similar ones. While the allowed region remains wide, the coexistence of the two species is limited to a narrow range of $r_{0 \mathrm{~B}}$.

by the sensitivity niche vectors:

$$
\mathcal{V}_{\boldsymbol{S}}=\left|\boldsymbol{S}_{1}\right|\left|\boldsymbol{S}_{2}\right| \sin \left(\boldsymbol{S}_{1}, \boldsymbol{S}_{2}\right)
$$

where $\left(\boldsymbol{S}_{1}, \boldsymbol{S}_{2}\right)$ denotes the angle between the vectors $\boldsymbol{S}_{1}$ and $\boldsymbol{S}_{2}$. It can be 
related to the scalar product

$$
\boldsymbol{S}_{1} \cdot \boldsymbol{S}_{2}=\sum_{l=1}^{D} S_{1 l} S_{2 l}
$$

of the niche vectors which is considered as the measure of "niche overlap" (Petraitis, 1989). The scalar product can be expressed (Anton, 1984, p. 184) as

$$
\boldsymbol{S}_{1} \cdot \boldsymbol{S}_{2}=\left|\boldsymbol{S}_{1}\right|\left|\boldsymbol{S}_{2}\right| \cos \left(\boldsymbol{S}_{1}, \boldsymbol{S}_{2}\right)
$$

so the volume and the niche overlap are related as

$$
\left(\mathcal{V}_{\boldsymbol{S}}\right)^{2}=\left|\boldsymbol{S}_{1}\right|^{2}\left|\boldsymbol{S}_{2}\right|^{2}-\left(\boldsymbol{S}_{1} \cdot \boldsymbol{S}_{2}\right)^{2}
$$

If the normalizations $\left|\boldsymbol{S}_{1}\right|$ and $\left|\boldsymbol{S}_{2}\right|$ are kept constant, the volume $\mathcal{V}_{\boldsymbol{S}}$ is small when the overlap of the sensitivity niches is large. A similar statement applies to the $\boldsymbol{C}$ vectors. Thus, a small overlap of both kinds of niche vectors is a requirement for robust coexistence.

The phenomenon is demonstrated for $L=D=2$ in Figure 4. The twodimensional regulating variable is

$$
\boldsymbol{I}=n_{\mathrm{A}} \boldsymbol{C}_{\mathrm{A}}+n_{\mathrm{B}} \boldsymbol{C}_{\mathrm{B}}
$$

where indices $\mathrm{A}$ and $\mathrm{B}$ are used to distinguish the two populations. As the biological interpretation requires $n_{\mathrm{A}}, n_{\mathrm{B}} \geq 0$, the wedge between the impact niche vectors $\boldsymbol{C}_{\mathrm{A}}$ and $\boldsymbol{C}_{\mathrm{B}}$ represents the possible environmental conditions. The sensitivity niche vectors point in the direction of steepest decrease of the growth rate of the corresponding population in the space of regulating variables. The environmental conditions satisfying the equilibrium equation of any of the two populations lie on a straight line, the Zero Net Growth Isocline (ZNGI, Tilman, 1982), which is perpendicular to the sensitivity niche vector of the corresponding population. The crossing point of the two ZNGIs is the equilibrium point. It must lie within the wedge of allowable environmental conditions to represent a biologically relevant coexistence of the two populations. If the equilibrium point lies outside the wedge, it corresponds to a (formal) solution of the equilibrium equations in which one of the population sizes is negative. In the panes (b-d) a series of equilibrium points, corresponding to a series of different values of $r_{0 \mathrm{~B}}$, is plotted. Observe, that only a very narrow range of the $r_{\mathrm{OB}}$ values allow coexistence if either the two impact niches [as in (c)], or the two sensitivity niches [as in (d)], are similar in direction.

\subsection{Niche overlap in case of a resource continuum}

The linear theory developed in the previous Sections applies for infinite dimensional regulation without essential modifications. As an example, we in- 
vestigate the text-book case of a resource continuum.

Suppose that some (e.g. bird) populations compete for seeds of different sizes. Denote the seed-size (more generally: the quality of the resource) by $q$. As $q$ is a continuous quantity, a change of notation is in order. While the depletion $I_{k}$ of the $k$ th resource was the $k$ th interaction variable in the finite dimensional case, depletion $I(q)$ of the resource of quality $q$ plays the same role for the case of resource continuum. The function $q \mapsto I(q)$ substitutes for the vector $\boldsymbol{I}$. The different notation does not reflect any conceptual difference between the finite and the infinite dimensional case as the finite dimensional vector $\boldsymbol{I}=\left\{I_{1}, I_{2}, \ldots, I_{D}\right\}$ can be seen also as a function $k \mapsto I_{k}$ defined on the set of $\{1,2, \ldots, D\}$.

Also the niche vectors become functions in the case of a resource continuum. For the $i$ th population, $C_{i}(q)$ and $S_{i}(q)$ measure the impact on, and the sensitivity towards, the resource of quality $q$, respectively. The linear impact and sensitivity maps can be written as

$$
I(q)=\sum_{j=1}^{L} C_{j}(q) n_{j}
$$

and

$$
r_{i}=r_{0 i}-\int S_{i}(q) I(q) d q \quad(i=1,2, \ldots, L)
$$

Then, again,

$$
r_{i}\left(\boldsymbol{E}, \mathcal{I}\left(n_{1}, n_{2}, \ldots, n_{L}\right)\right)=r_{0 i}(\boldsymbol{E})-\sum_{j=1}^{L} a_{i j} n_{j}
$$

for each $i$, where now the elements of the community matrix are given by

$$
a_{i j}=\int S_{i}(q) C_{j}(q) d q \quad(i, j=1,2, \ldots, L) .
$$

For the continuous case the niche overlap is measured by the "overlap integral" of the niche functions, which are the infinite dimensional analogues of the scalar product (23):

$$
\begin{aligned}
\boldsymbol{S}_{i} \cdot \boldsymbol{S}_{j} & =\int S_{i}(q) S_{j}(q) d q, \\
\boldsymbol{C}_{i} \cdot \boldsymbol{C}_{j} & =\int C_{i}(q) C_{j}(q) d q .
\end{aligned}
$$

The theory of the previous sections applies: The higher the niche overlap is, the smaller the parameter range allowing coexistence. We emphasize again that both the impact and the sensitivity niche functions must differ, in the sense of small overlap, for robust coexistence. 
We may arrive at the intuitive notion of the resource utilization function (MacArthur \& Levins, 1967), if we suppose that the impact and the sensitivity niche functions are proportional (cf. Petraitis, 1989):

$$
C_{i}(q)=\alpha S_{i}(q)
$$

Then, the resource utilization function can be defined as

$$
u_{i}(q)=\sqrt{\alpha} S_{i}(q)=\frac{1}{\sqrt{\alpha}} C_{i}(q)
$$

With this identification, the community matrix (i.e., the competition coefficients for resource competition) assumes the usual form

$$
a_{i j}=\int u_{i}(q) u_{j}(q) d q
$$

i.e., it is the overlap of the two resource utilization functions. The small overlap is the usual condition for robust coexistence (cf. May, 1973, 1974).

The notion of the resource utilization function expresses the intuition that a population affects, and depends on, the resources that it uses. However, there is no strong biological basis for the exact proportionality expressed by Eq. (33). It should be considered as no more than a convenient simplifying assumption that can be removed with little effort. Yodzis (1989, p. 119) provides a mechanistic underpinning of Eq. (35) by explicit modeling of the resource dynamics. However, the emerging resource utilization function is an artificial combination of factors, lacking clear biological content. It is easy to repeat that derivation in a more natural way in terms of the impact and the sensitivity functions.

\subsection{Structural instability of Roughgarden's example}

Non-robustness of coexistence of similar populations hints that the coexistence of a continuum of populations cannot be robust, either. Following Gyllenberg \& Meszéna (2005), here we show the Roughgarden's (1979) example for continuous coexistence is structurally unstable.

The Lotka-Volterra type population regulation [Eq. (29)] can be rewritten for the continuous density distribution as

$$
r(y)=r_{0}(y)-\int a(y, x) n(x) d x .
$$

Here, $r(y)$ and $r_{0}(y)$ are the growth rate and the intrinsic growth rate of trait $y$, respectively. The distribution of the populations along the trait axis $x$ is 

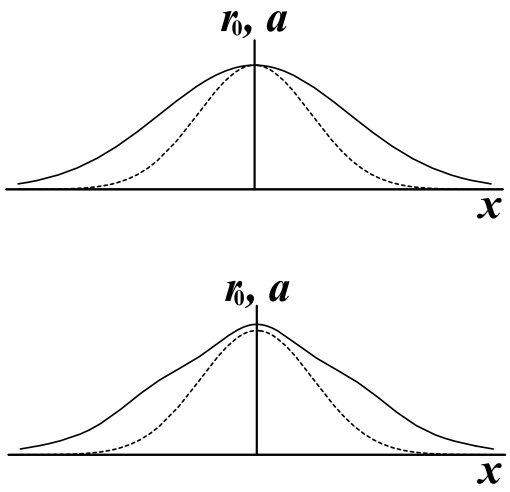

$\mathbf{a}$

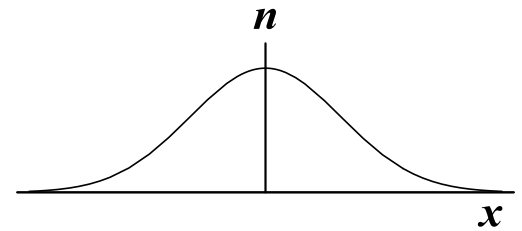

b

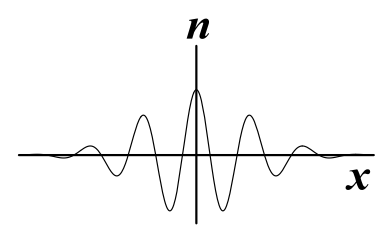

Fig. 5. Structural instablity of Roughgarden's (1995) model for continuous coexistence. Left plots: the intrinsic growth rate $r_{0}(x)$ (solid line) and the competition kernel $a(x)$ (dotted line). Right plots: the equilibrium population density, as calculated on the whole real line by de-convolution. (a) A Gaussian $r_{0}$ allows coexistence of a continuum of the populations. (b) Periodic perturbation of $r_{0}$ according to Eq. (40). The equilibrium density experiences a high perturbation and looses its positivity in some intervals.

described by the density function $n(x)$. As Roughgarden noted, the Gaussian choices

$$
r_{0}(x)=\frac{1}{\sqrt{2 \pi} w} e^{-\frac{x^{2}}{2 w^{2}}}
$$

and

$$
a(y, x)=\frac{1}{\sqrt{2 \pi} \sigma} e^{-\frac{(y-x)^{2}}{2 \sigma^{2}}}
$$

allows the continuous distribution

$$
n(x)=\frac{1}{\sqrt{2 \pi\left(w^{2}-\sigma^{2}\right)}} \cdot e^{-\frac{x^{2}}{2\left(w^{2}-\sigma^{2}\right)}}
$$

of populations to coexist in equilibrium (Figure 5a).

However, a small periodic perturbation of the intrinsic growth rate

$$
r_{0}^{\prime}(x)=r_{0}(x)(1+\varepsilon \cos k x),
$$

where $\varepsilon$ is the amplitude and $k$ is the "frequency" of the perturbation, results in a widely oscillating solution (Figure 5b):

$$
n^{\prime}(x)=\frac{1}{\sqrt{2 \pi\left(w^{2}-\sigma^{2}\right)}} e^{-\frac{x^{2}}{2\left(w^{2}-\sigma^{2}\right)}}\left[1+\varepsilon e^{+\frac{w^{2} \sigma^{2}}{2\left(w^{2}-\sigma^{2}\right)} k^{2}} \cos \left(\frac{w^{2}}{w^{2}-\sigma^{2}} k x\right)\right] .
$$

Obviously, for any $\varepsilon$, one can choose the perturbation frequency $k$ so large that the perturbed equilibrium (41) will not remain everywhere non-negative. In this case, the solution (41) no longer describes a biologically meaningful 
coexistence. Therefore, the coexistence of all populations along the trait axis can be destroyed by an arbitrarily small perturbation by giving relative advantage/disadvantage to very similar populations.

\section{The general theory}

\section{$4.1 \quad$ Non-linear generalizations}

In this section we demonstrate how the conclusions of the linear theory above generalizes to the non-linear case. For this purpose, we define the two kinds of niches and the community matrix as the derivatives of the (possibly non-linear) functions $\mathcal{I}, \mathcal{S}$ and $\mathcal{R}$. We demonstrate that the volume measures depicted in Figure 3 are still usable.

The impact niche vector is defined as the derivative of the impact map $\mathcal{I}$ :

$$
\boldsymbol{C}_{j}=\frac{\partial \boldsymbol{I}}{\partial n_{j}} \quad(j=1,2, \ldots, L) .
$$

It measure the impact of a small change of the population size. Obviously, this "differential" impact depends on the population sizes at which the derivative is taken. We suppress this dependence in the notation. Similarly, the (differential) sensitivity niche vectors are defined as

$$
\boldsymbol{S}_{i}=-\frac{\partial r_{i}}{\partial \boldsymbol{I}} \quad(i=1,2, \ldots, L)
$$

For finite dimensional population regulation, the derivatives (42) and (43) can be written component-wise as partial derivatives

$$
C_{j k}=\frac{\partial I_{k}}{\partial n_{j}} \quad(k=1,2, \ldots, D ; j=1,2, \ldots, L)
$$

and

$$
S_{i k}=-\frac{\partial r_{i}}{\partial I_{k}} \quad(k=1,2, \ldots, D ; i=1,2, \ldots, L),
$$

representing the two-way interaction between the $i$ th population and the $k$ th regulating variable. In a similar manner, if the environmental interaction is captured by a function, like in Section 3.4, then the two kinds of niche are specified by the functions $C_{i}(q)$ and $S_{i}(q)$ representing the local per capita impact on, and the sensitivity to, the regulating variable $I(q)$. Boxes 1 and 2 provide examples for calculating the niche vectors with pure resource competition and with joint regulation via competition and predation, respectively. 
The (differential) community matrix is defined as the derivative of the population regulation $\mathcal{R}$ :

$$
a_{i j}=-\frac{\partial r_{i}}{\partial n_{j}} \quad(i, j=1,2, \ldots, L) .
$$

Again, its dependence on $n_{j}$ is suppressed in the notation. The element $a_{i j}$ measures the sensitivity of the growth rate of the $i$ th population to the size of the $j$ th population. The chain rule for derivatives establishes the same connection between the differential niche vectors and the differential community matrix as in the linear model:

$$
a_{i j}=\boldsymbol{S}_{i} \cdot \boldsymbol{C}_{j}
$$

As known from multivariate calculus (Apostol, 1962, pp. 84-86, Edwards, Jr., 1973, p. 245), the function $\mathcal{R}$ maps the infinitesimal volume element $d n_{1} d n_{2} \ldots d n_{L}$ around the point $\left(n_{1}, n_{2}, \ldots, n_{L}\right)$ into a parallelepiped of volume $|J| d n_{1} d n_{2} \ldots d n_{L}$, where $J=\operatorname{det} \boldsymbol{a}$ is the determinant of the community matrix evaluated at that point. That is, in complete analogy with the linear case, $|J|$ is the right measure of the local strength of population regulation on the community level. If $|J|$ is small, the growth rates depend weakly on the population sizes at least in some directions (Figure 6). The impact function $\mathcal{I}$ maps the volume element $d n_{1} d n_{2} \ldots d n_{L}$ into a parallelepiped of volume $\mathcal{V}_{\boldsymbol{C}} d n_{1} d n_{2} \ldots d n_{L}$. Accordingly, the volume $\mathcal{V}_{\boldsymbol{C}}$ is the measure of the strength of the environmental impact of the populations. Analogously, the volume $\mathcal{V}_{\boldsymbol{S}}$ measures of the environmental sensitivity of the populations.

As a direct consequence of relation (47), Proposition of Section 3.3 remains valid. Accordingly, either weak environmental impact, or weak environmental dependence lead to weak population regulation. With this connection between the community matrix and niche in place, generalization of the linear result is established if we demonstrate the detrimental effect of small $|J|$ on the robustness of coexistence.

An implicit differentiation of the set of equilibrium equations

$$
r_{i}\left(\boldsymbol{E}, \mathcal{I}\left(n_{1}, n_{2}, \ldots, n_{L}\right)\right)=0 \quad(i=1,2, \ldots, L)
$$

gives the sensitivity of the equilibrium population sizes towards the external parameters:

$$
\frac{\partial n_{i}}{\partial \boldsymbol{E}}=\sum_{j=1}^{L} a_{i j}^{-1} \frac{\partial r_{j}}{\partial \boldsymbol{E}}=\frac{1}{J} \sum_{j=1}^{L} \operatorname{adj}(a)_{i j} \frac{\partial r_{j}}{\partial \boldsymbol{E}}
$$

provided that $J \neq 0$.

Clearly, the equilibrium densities become extremely sensitive to the external parameters whenever $|J|$ becomes small. As the population sizes must remain 

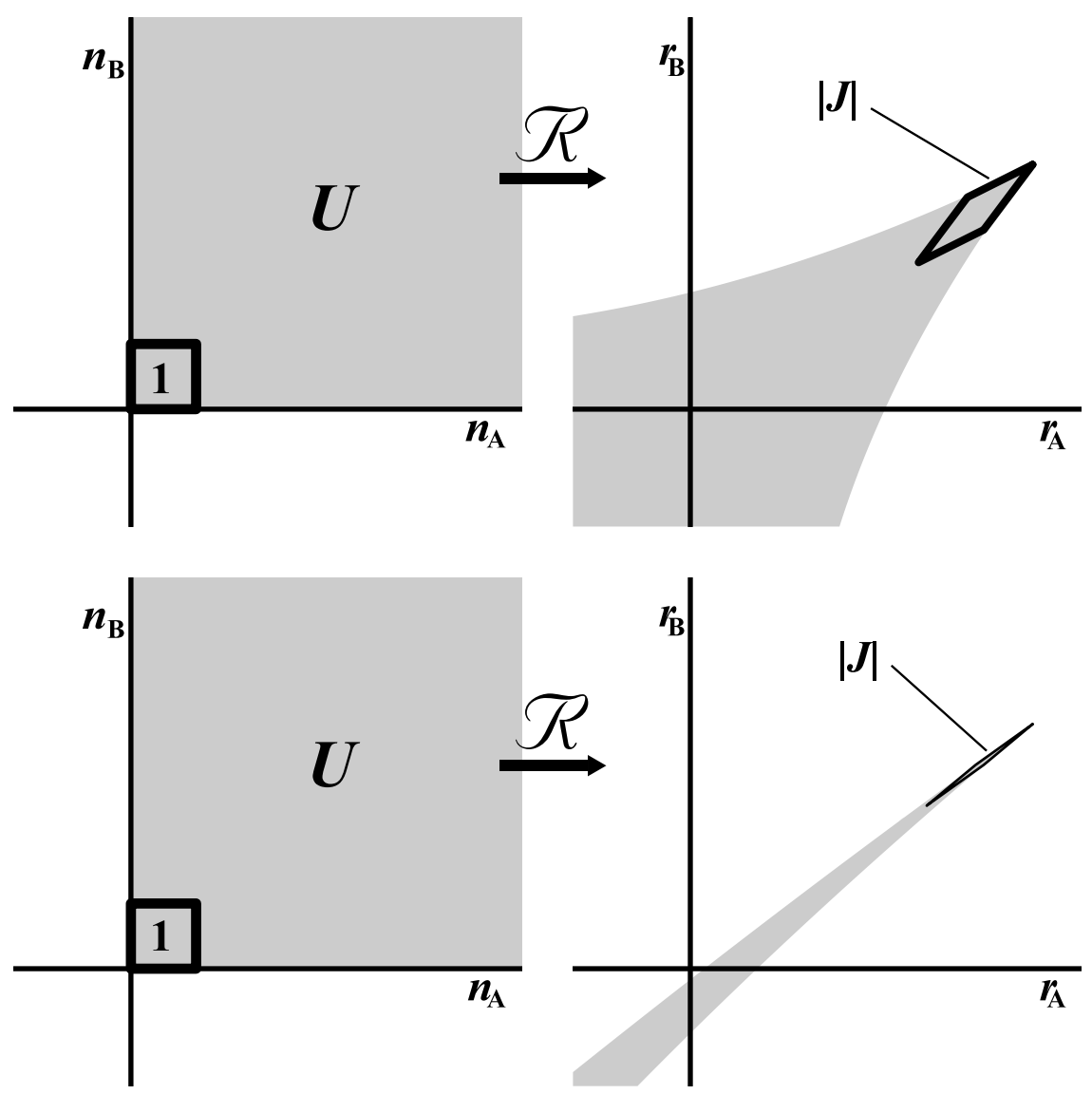

Fig. 6. Coexistence of two populations with non-linear population regulation. As in Figure 2, the left panes represent the space of population sizes while the right panes represent the space of growth rates of the populations. The gray areas denote the possible (positive) population sizes on the left panes and the corresponding growth rates on the right panes. Because of the non-linearity, the unit box should be chosen small enough. Then, the image of the box is still a parallelogram with area $|J|$. On the top row, coexistence is possible, because the zero growth rates (the origin at the right pane) is in the allowed region. On the bottom row, this condition is not fulfilled and no coexistence is possible. Here, $|J|$ is small everywhere, so the allowed region is narrow. Fine-tuning of the model parameters would be needed to arrange coexistence.

bounded, this sensitivity will result in extinction of one of the populations even for a relatively small change of the external parameters. As demonstrated in Figure 6 , in the case of small $|J|$, an extreme fine-tuning of the parameters is needed to effect coexistence. 


\section{Box 1: Impact and the sensitivity in Tilman's model}

In a model of Tilman (1982), which is also the basis of the niche theory of Leibold (1995) and Chase \& Leibold (2003), $L$ species compete for $D$ resources. In the most general version, the dynamics is specified as

$$
\frac{1}{n_{i}} \frac{d n_{i}}{d t}=r_{i}=f_{i}\left(R_{1}, R_{2}, \ldots, R_{D}\right)-m_{i}
$$

and

$$
\frac{d R_{j}}{d t}=g_{j}\left(R_{j}\right)-\sum_{i=1}^{L} n_{i} f_{i}\left(R_{1}, R_{2}, \ldots, R_{D}\right) H_{i j}\left(R_{1}, R_{2}, \ldots, R_{D}\right)
$$

(Tilman, 1982, p. 14). Here, $R_{j}$ is the availability of resource $j ; m_{i}$ is the mortality rate of species $i ; f_{i}$ is the function describing the dependence of the growth rate of species $i ; H_{i j}$ is the function describing the amount of resource $j$ required to produce each new individual of species $i$. In the more specific version of the model,

$$
g_{j}\left(R_{j}\right)=a\left(R_{j}^{0}-R_{j}\right),
$$

where $a$ is a constant, and the complicated dependence for per-capita resource consumption $f_{i}\left(R_{1}, R_{2}, \ldots, R_{k}\right) H_{i j}\left(R_{1}, R_{2}, \ldots, R_{k}\right)$ is replaced by the constant $c_{i j}$ (Tilman, 1982, p. 76). Then, the resource dynamics becomes linearized, as

$$
\frac{d R_{j}}{d t}=a\left(R_{j}^{0}-R_{j}\right)-\sum_{i=1}^{L} n_{i} c_{i j}
$$

At fixed densities, the resource equilibrium point can be calculated as

$$
R_{j}^{*}=R_{j}^{0}-\frac{1}{a} \sum_{i=1}^{L} n_{i} c_{i j}
$$

Then, the regulating variable, i.e., the resource depletion, is

$$
I_{j}=R_{j}^{0}-R_{j}^{*}=\frac{1}{a} \sum_{i=1}^{L} n_{i} c_{i j}=\sum_{i=1}^{L} n_{i} C_{i j},
$$

where components of the impact niche vector, as we defined, are

$$
C_{j i}=\frac{c_{i j}}{a} .
$$

Our sensitivity niche vector is

$$
S_{i j}=-\frac{\partial r_{i}}{\partial I_{j}}=\frac{\partial f_{i}}{\partial R_{j}}
$$

This gradient vector is obviously perpendicular to the $r=$ const. isoclines, including the ZNGI, corresponding to $r=0$.

\subsection{Probability of coexistence}

On assuming a probability distribution for the external parameters we can actually calculate the probability of conditions allowing the coexistence. 
For the sake of simplicity of the derivation, suppose that the effect of the external parameters on the $i$ th population is represented by the excess mortality $\Delta_{i}(i=1,2, \ldots, L)$. Accordingly, we write the dependence on the external variables in the form

$$
r_{i}\left(\boldsymbol{E}, \mathcal{I}\left(n_{1}, n_{2}, \ldots, n_{L}\right)\right)=\hat{r}_{i}\left(\mathcal{I}\left(n_{1}, n_{2}, \ldots, n_{L}\right)\right)-\Delta_{i}(\boldsymbol{E}) .
$$

(Negative $\Delta_{i}$ represents decreased mortality or increased birth rate.) Then the equilibrium equations become

$$
\hat{r}_{i}\left(\mathcal{I}\left(n_{1}, n_{2}, \ldots, n_{L}\right)\right)=\Delta_{i}(\boldsymbol{E}) \quad(i=1,2, \ldots, L) .
$$

We collect the variables $n_{i}, \hat{r}_{i}$ and $\Delta_{i}$ into the vectors $\boldsymbol{n}, \hat{\boldsymbol{r}}$ and $\boldsymbol{\Delta}$. The probability distribution of the environmental parameters translate to a (supposedly continuous) probability distribution of the excess mortalities, represented by the probability density $p(\boldsymbol{\Delta})$. Then the probability $P(\boldsymbol{n}>0)$ of having all equilibrium densities positive satisfies

$$
P(\boldsymbol{n}>0)=\int_{\boldsymbol{n}>0} \frac{p[\hat{\boldsymbol{r}}(\boldsymbol{n})]}{k[\hat{\boldsymbol{r}}(\boldsymbol{n})]}|J(\boldsymbol{n})| d \boldsymbol{n},
$$

where $k[\varrho]$ denotes the number of all positive $\boldsymbol{n}$ values for which $\varrho=\hat{\boldsymbol{r}}(\boldsymbol{n})$. (See Stomberg, 1981, p. 385 for $k=1$ and Rado \& Reichelderfer, 1955, p. 363 for the the general case.)

It is clear from Eq. (52) that the probability that all $L$ species coexist goes to zero when $|J(\boldsymbol{n})| \rightarrow 0$ for all relevant $\boldsymbol{n}$.

We stress that this probabilistic argument does not assume any stochasticity in the behavior of the populations. We calculated the probability of finding the proper conditions for coexistence in a randomly chosen location. A community with small $|J|$ has a small chance to find the permitting combinations of the external parameters. 


\section{Box 2: Keystone predator}

Here we use the "keystone predator" model of Chase \& Leibold (2003, p. 49) to demonstrate the applicability of our concepts in situations other than resource competition. Coexistence of two species (abundances: $N_{1}$ and $N_{2}$ ), that are regulated by a single resource $R$ and a single predator $P$, is investigated. The dynamics is specified as

$$
\begin{aligned}
& \frac{d R}{d t}=c[S-R]-f_{1} N_{1}-f_{2} N_{2} \\
& \frac{d N_{i}}{d t}=\left(f_{i} a_{i} R-m_{i} P-d_{i}\right) N_{i} \quad i=1,2 \\
& \frac{d P}{d t}=\left(m_{1} c_{1} N_{1}+m_{2} c_{2} N_{2}-d_{\mathrm{P}}-\alpha P\right) P,
\end{aligned}
$$

where the coefficients are self-explanatory. The vector of regulating variables is $\boldsymbol{I}=(R, P)$. (As we have variables other, than resources, we no longer stick ourselves to the "exploitation" interpretation and sign convention.) The two regulating factors allow coexistence of at most two species on the trophic level of interest. We consider the predator as a part of the environment, instead of a species of our interest. We allow it to be regulated by factors other than dependence on "our" species. The term $-\alpha P$ collects all the extra regulations. The equilibrium values of the regulating variables are

$$
\begin{aligned}
& R^{*}=S-\frac{1}{c}\left[f_{1} N_{1}+f_{2} N_{2}\right] \\
& P^{*}=\frac{1}{\alpha}\left[m_{1} c_{1} N_{1}+m_{2} c_{2} N_{2}-d_{\mathrm{P}}\right] .
\end{aligned}
$$

Both the impact and the sensitivity vectors have an " $\mathrm{R}$ " and a "P" component:

$$
\begin{aligned}
C_{i \mathrm{R}} & =\frac{\partial R^{*}}{\partial N_{i}}=-\frac{f_{i}}{c} & C_{i \mathrm{P}} & =\frac{\partial P^{*}}{\partial N_{i}}=\frac{m_{i} c_{i}}{\alpha} \\
S_{i \mathrm{R}} & =-\frac{\partial r_{i}}{\partial R}=-f_{i} a_{i} & S_{i \mathrm{P}} & =-\frac{\partial r_{i}}{\partial P}=m_{i}
\end{aligned}
$$

This description breaks down for $\alpha=0$, i.e., when the investigated populations are the only regulators of the predator. Then, there is no such thing, as equilibrium predator density at a given values of $N_{1}$ and $N_{2}$, because the predator grows exponentially at fixed $N_{i}$. In this case, we have to include the predator into the list of the populations of interest and introduce new regulating variables to parametrize their interactions.

Our findings can be summarized as follows.

\section{Limiting Similarity Principle}

Any of the following conditions imply the next one and make coexistence of a given set of populations improbable, i.e., restricted to a narrow range of the external environmental parameters:

- Large overlap between either the impact or the sensitivity niches.

- Small $\mathcal{V}_{\boldsymbol{C}}$ or $\mathcal{V}_{\boldsymbol{S}}$.

- Small $|J|$. 
The Discrete Competitive Exclusion Principle is a limiting case. If its condition $L \leq D$ is violated then $L$ number of $D$ dimensional vectors cannot be linearly independent, implying $\mathcal{V}_{\boldsymbol{C}}=\mathcal{V}_{\boldsymbol{S}}=|J|=0$ and structural instability of coexistence.

\section{Discussion}

\subsection{Competitve exclusion and limiting similarity}

The "competitive exclusion principle" is a controversial, but central, reference point in community ecology. By the "admittedly unclear" words of Hardin (1960), the principle is stated as "complete competitors cannot coexist"; or, "ecological differentiation is the necessary condition for coexistence". We removed the ambiguity by a mathematical characterization of the nature and extent of the required differentiation. "Limiting similarity" is a robust prediction in a well-defined sense. While there is no absolute lower bound for the permitted similarity, coexistence of similar populations is possible only for a narrow range of external parameters. Although, the robustness/probability of coexistence of a given set of populations can be calculated only in a specific model context, the idea of shrinking possibility of coexistence with increasing similarity is general.

May \& MacArthur (1972) and May $(1973,1974)$ concluded that strong enough population regulation, therefore limiting similarity, was needed to compensate for the effect of noise and to avoid the extinction of some of the populations. While this conclusion is fully consistent with our analysis, Rosenzweig (1995, p. 127) interpreted May's results as an early recognition of the lack of limiting similarity in a constant environment. We emphasize that limiting similarity is the expectation even in a constant environment.

As limiting similarity is the general expectation, it is of no surprise that the continuous coexistence in Roughgarden's (1979) example is structurally unstable, as we demonstrated in Section 3.5. Lack of structural stability of Roughgarden's model was seen already implicitly by Sasaki \& Ellner (1995), Sasaki (1997) when they investigated unrelated but mathematically isomorphic models. (We thank Yoh Iwasa for pointing to this connection. See also Haccou \& Iwasa, 1995, 1998.) Szabó \& Meszéna (submitted) analyzed Roughgarden's model beyond the scope of small perturbations studied here. They demonstrated the generic validity of the usual "limiting similarity" expectations with respect to the Lotka-Volterra model. The possibility, but exceptionality, of continuous coexistence was noted also by Geritz $(1995,1999)$ for asymmetric seed competition, by Kitzing et al. (1999) in metapopulation context and by 
Meszéna \& Szathmáry (2001) for prebiotic replicators. Gyllenberg \& Meszéna (2005) reviewed the issue and proved a very general statement: Coexistence of infinitely many populations in a finite volume of the phenotype space is structurally unstable for any model constructed from smooth ingredients.

\subsection{Connection to the dynamics of adaptation}

Ecological conditions for coexistence are related to frequency-dependent selection (Christiansen, 1988; Heino et al., 1998). Accordingly, both the general impossibility and the exceptional possibility of coexistence of similar strategies are of evolutionary relevance. A general theory of frequency-dependent selection (Metz et al., 1996; Geritz et al., 1997, 1998; Meszna et al., in press) demonstrates that similar phenotypes can coexist only at the "singular" points of the phenotype space, where the selection gradient vanishes. Competitive exclusion, i.e., the "survival of the fittest", operates away from the singular points in line with the usual picture of natural selection (see also Dieckmann \& Law, 1996, for directional evolution in this context). On the other hand, the possibility of coexistence in the vicinity of singular points allows the "branching" type evolution, which was suggested to be related to speciation (Dieckmann \& Doebeli, 1999; Doebeli \& Dieckmann, 2003; Kisdi \& Geritz, 1999; Geritz \& Kisdi, 2000; Meszéna et al., 1997; Mizera \& Meszéna, 2003; Schreiber \& Tobiason, 2003; Geritz et al., 2004). This way, although coexisting species generically must differ considerably, and live in different niches, a new species can emerge via small modifications. (See Meszéna \& Metz, 1999 for the specific connection between the current theory, frequency-dependent selection and speciation.)

\subsection{Population regulation and coexistence}

Regulation of a single population and coexistence of several populations are usually discussed separately. We offered a unifying viewpoint. Robust coexistence, i.e., the lack of competitive exclusion, is equivalent to the existence of separate population regulation for each population, i.e., significant differences both in the way the populations affect the regulating variables and in the way the population growth rates depend on these variables. Without such differentiation, competitive exclusion, i.e., the impossibility of coexistence, is the default relation between two populations.

Competitive exclusion is a relatively fast process, except when $r_{1} \approx r_{2}$. (Here, $r_{1}$ and $r_{2}$ denote the growth rates of the two species involved.) According to Eq. (5), the time-scale of the competitive exclusion is $1 /\left(r_{1}-r_{2}\right)$. For instance, a permanent $1 \%$ difference either in the birth or in the death rates results in competitive exclusion on the approximate time-scale of 100 generations. 
Equality of the demographic parameters of two different species, without a specific reason, is highly unlikely. Either the two species have to be truly identical in all ecological and demographic respects, or the equality of the growth rates has to be established by a regulating feedback. As examples for the first case, sibling species (Turner, 1999) may be able to coexist without niche segregation. (Loss of one of the two identical species via demographic stochasticity can be extremely slow when the population sizes are large enough.) However, as soon as discernible differences - other than in their mating traits - arise between the species, coexistence requires that their fitness difference is made zero by differential regulation (Galis \& Metz, 1998). In this paper we are concerned with the latter type of regulated (or stable, Chesson, 2000b) coexistence. See Goldschmidt et al. (1990), Seehausen \& Bouton (1997), Bouton et al. (1997) for detailed investigations of niche segregation, i.e., the emergence of differential regulation, between recently formed Cichlid species.

Chesson (1994, 2000ab) investigated the coexistence-stabilizing mechanisms via comparing the growth rates of the populations, when rare, and when they are in equilibrium: rarity must provide an advantage. In contrast, our approach tests the effect of small abundance-changes on the growth rates. The extra complication of our differential approach pays off in the linearity of the perturbation and in allowing to reach the limiting similarity conclusion. Actually, our results rely on a condition weaker than dynamical stability. Structural stability (robustness) requires det $\boldsymbol{a}$ to be non-zero (large enough in absolute value), while linear dynamical stability is based on the stronger condition of negative real part of all eigenvalues of the population matrix $\boldsymbol{a}$.

By now, a consensus has been reached on the traditionally "controversial" (Krebs, 2001, p. 290) issue of population regulation. It has been established that any population has to be regulated (Turchin, 1999, 2003; Hanski, 1999). However, as the notion of regulating factors are not recognized in community ecology in their generality, controversies about competitive exclusion persist (cf. den Boer, 1986; see Gorskov et al. 2000 for a consistent picture, different from the ours). It is a general view that competitive exclusion and niche are relevant issues only for communities shaped by resource competition (Begon et. al., 1996, p. 265). It is a widely accepted argument that "... there are many situations, where disturbance, such as predation, storms on a rocky shore or frequent fires, may hold down the densities of populations, so that the resources are not limiting and the individuals do not compete for them." (Begon et al., 1996, p. 802). Then, it is assumed that reduced competition reduces the danger of competitive exclusion and increases diversity beyond what is permitted by "competition" (Houston, 1979, Begon et al., 1996, p. 802).

This type of reasoning is misleading. There is no essential difference between resource competition and the other types of population regulation, like pre- 
dation and disease spread (c.f. Case \& Leibold, 2003). Resources are not limiting/regulating only when other factors regulate. Then, the structure of that regulation has to be studied in order to predict the effect on diversity. For instance, in the simplest case, a single predator contributes a single regulating factor, so it can allow one, and only one, additional species to coexist (see Box 2). In particular, suggestion that "disturbance" slows down competitive exclusion (see Huston, 1979, 1994) is baseless, as disturbance does not habitually decreases the difference between the growth rates.

Because of the propensity of the regulating factors to be high in number, we need a way to count only the really different ones. In line with Abrams (1988), our version of Limiting Similarity Principle in Section 4 provides the necessary background: the limited overlap of the niches, the impact ones as well as the sensitivity ones, is the criterion (see also Meszéna \& Metz, 1999). This way we unified the discrete and the continuous versions of the theory of competitive exclusion.

\subsection{Spatially extended populations}

Beyond being fully identical, an extremely strong migration barrier between the populations is the only mathematical possibility to avoid the necessity of regulated coexistence: If the migration between two habitats is negligible even on the evolutionary time-scale then there is no danger of competitive exclusion between the species living separately.

Else, the populations equilibrates between the habitats on the evolutionary time-scale. Then, our theory applies if we identify the $r_{i}$ s with the overall population growth rates after the spatial relaxation. The overall rates are the averages of the local ones, weighted by the relaxed local abundances (Chesson, 2000a).

The theory of structured populations (Metz \& Diekmann, 1986; Caswell, 2000; Diekmann et al., 1998, 2001, 2003) applies for calculating the $r_{i}$ s as well as the equilibrium distributions. In this paper we are interested in either the equilibrium of the populations or small perturbations of this equilibrium. Then the analysis of Greiner et al. (1994) establishes the time-scale separation between the slow overall growth/decline of the population and the faster spatial relaxation. (Here we assume a finite world with bounded migration time-scale.) Populations, which are structured in any other respect, can be studied similarly.

When a spatially extended population is considered, the overall $\boldsymbol{E}$ and $\boldsymbol{I}$ vectors should include the values of the external parameters and the regulating variables at each location, respectively (cf. the variables $E_{j x}$ and $C_{j x}$ of Ches- 
son, 2000a). For instance, in the two patch case of Meszéna et al. (1997) the population is regulated by a single factor in both patches separately. Then, $\boldsymbol{I}$ is the two-dimensional vector of the two local regulating variables. The analogous situation for an environmental gradient is studied by Czárán (1989), Mizera \& Meszéna (2003), Mizera et al. (in prep). Here the regulation is described by a continuum of regulating variables, i.e., by a location-dependent regulator. An important conclusion of these studies is, that the environmental tolerance and the mobility of the competitors together determine the extent of the region occupied by a single species.

In general, even a single resource can act as a continuum of regulating factors along an environmental gradient. The environmental condition at the location of the resource should be considered as a quality of that resource. Examples include the light gradient (Hutchinson 1961) or the temperature gradient (Tilman et. al. 1981) in a water volume, or the feeding space separation of warblers (MacArthur, 1958).

\subsection{Fluctuating environment}

Spatio-temporal fluctuations lead to increased diversity easily. As Armstrong \& McGehee (1976), Hsu et al. (1977) demonstrated, it is possible that two species feeding on the same resource can coexist robustly if the dynamical attractor is a periodic orbit. Adler (1990), Loreau (1989, 1992), Loreau \& Ebenhöh (1994), Huisman \& Weissing (1999) provided further examples for increased diversity via temporal fluctuations, internal or external. Czárán \& Bartha (1989, 1992), Czárán (1998) investigated diversity arising from complex spatio-temporal patterns. Tél et al. (2000), Károlyi et al. (2000, 2002), Szabó et al. (2002), Scheuring et al. (2003) studied coexistence in chaotic flows.

Still, as a non-regulated population fluctuates around a path of exponential growth/decline (Tuljapurkar, 1990; Metz et al., 1992; Ferrière \& Gatto, 1995), spatio-temporal heterogeneity does not weaken the necessity of having regulating/stabilizing mechanisms to equalize the long-term growth rates (Chesson, 1991; Hanski, 1999, p. 29). While competitive exclusion may be irrelevant locally, if a species is going to survive longer than the life-span of a single habitat, their regulation and competitive exclusion has to be considered globally, on a metapopulation level (Tilman et al., 1994; Parvinen \& Meszéna, in prep.).

Chesson (1994, 2000b) distinguish between two types of fluctuation-induced stabilizing mechanisms: "storage effect" and "effect of relative non-linearity". The first one essentially corresponds to temporal niche-segregation allowed by the fluctuation (cf. Christiansen \& Fenchel, 1977, p. 69), while the second one means that the descriptors of the fluctuation (like variance) emerges as 
additional regulating variables via the non-triviality of non-linear averaging (cf. Levins, 1979; Kisdi \& Meszéna, 1993).

These results make extremely plausible that our picture of decreasing robustness with increasing similarity can also be extended to fluctuation-mediated coexistence in stationarily fluctuating environments. However, this is beyond the scope of the current paper.

\subsection{Niche space as a map for competitive exclusion}

According to Hutchinson's (1978, p. 152-157) historical analysis, the notion of niche acquired its connection to Gause's principle gradually. While the relation between the niche overlap and the strength of competition is considered as the "central tenet of niche theory" (Case, 2000, p. 368), the actual usage of the term is not always consistent with this tenet (Juhász-Nagy, pers. comm.). For the consistency, the niche space has to be equated with the set of regulating factors/variables. This set can be either a finite/discrete one, or a continuum. In the latter case, the niche space is an Euclidean space of the "niche dimensions", which parametrize the continuum of the regulating factors (e.g. the resource quality $q$ in Sections 3.4 and 3.5). The niche of a species is given by specifying its impact on, and sensitivity towards, the regulating factors, a direct generalization of the notion of the resource utilization function. Such description of the niche of a species consists of two finite dimensional vectors, or two functions, depending on the finite or continuous nature of the niche space. We established that small overlap between both of the impact and of the sensitivity niche vectors/functions, i.e., the proper separation in the niche space, is the general prerequisite for robust coexistence.

Note that the niche space is not a pre-existing emptiness, which is filled out by the species later. Any new species may extend the niche space by contributing additional regulating factor(s).

Hutchinson's imagination was captured by the continuous case. He distinguished between two types of niche dimensions: resource quality ("bionomic") and environmental condition ("scenopoetic") (Hutchinson, 1978, p. 171). The Gauseian idea behind this distinction is clear. Reduced competition between two species can arise either by differing in local resource use or by living separately; the latter possibility is related to the different requirements of the species with respect to the ecological conditions. The connection between niche segregation and reduced competition is direct in the first case, but mediated via spatial distributions in the second one. Not accidentally, efforts of modeling niche segregation usually refer to a niche dimension of resource quality type (MacArthur, 1967; Yodzis, 1989, p. 119). Our picture unifies these two 
kinds of niche dimensions by recognizing that environmental conditions can be seen also as a quality of the resource (Section 5.4). (See also Christiansen \& Fenchel, 1977, p. 69.)

While, undoubtedly, Gause's principle had a central role in Hutchinson's thinking, his description of the niche as the "hypervolume" in the space of "all variables that affect a particular species" was too vague to secure the connection. Following this definition, environmental conditions are often regarded as the typical niche variables without any reference to population regulation. It was even suggested that considering resource quality as a niche dimension had been a logical mistake of Hutchinson. According to this view, the abundance, instead of the quality, of the food is the "right" niche dimension, as this is the "condition" type quantity (Leibold, 1995). However, the central tenet is lost in this way. Living under the same environmental conditions does not imply competitive exclusion, resource abundances counted, or not. Note that resource partitioning is a partitioning of the space of the resource qualities and not of the space of resource abundances. (On the other hand, the supply rate of a resource is a proper "condition" type niche dimension; Kőszeghy, 2004.) See also Begon et al. (1996), who introduces the notion of niche with the pure "condition" interpretation on p. 87, but stress that the concept is "most powerfull" in resource context, while they remain undecided about the quality-or-abundance issue on p. 130.

Hutchinson's distinction (p. 161) between the fundamental and the realized niche can be represented in our framework naturally by evaluating the defining derivatives (42) and (43) in the absence and in the presence of the competitors, respectively.

The possibility of a niche "space" consisting of a finite number of points seems to contradict radically the "hypervolume" notion of Hutchinson. However, from a biological, as opposed to a formal, point of view, this is the right picture for the discrete case. If the populations are limited by a finite number of resources, partitioning of this finite set of resources is the proper analogy to the partitioning of the resource quality continuum. The discrete notion of niche, as a finite dimensional vector, was recognized already by Petraitis (1989).

The discrete version of the resource competition theory was rejuvenated by Tilman (1982). He emphasized the need for "mechanistic" modeling, i.e., for explicit representation of the feedback loop. Leibold (1995), Chase \& Leibold (2003) re-interpreted the notion of niche in the context of Tilman's model. Their approach has strong similarities to the one which was pursued in this paper:

- They stress the importance of regulating factors other than resources, espe- 
cially the regulating role of predators.

- They stress the importance of considering the two legs of regulation: the population's impact and dependence on the regulating factors.

Our notion of the impact niche, i.e., the per capita effect $\boldsymbol{C}$ on the regulating variables, is very similar to Leibold's one, i.e., the per capita consumption rate c. (See Box 1 for the explicit connection between the two. The slight difference was motivated by the generality of our choice.) Leibold's "requirement" niche of a species is the region of the hyperspace of the abundances of the finite number of resources (or other regulating factors). Instead, we proposed the "sensitivity" niche vectors in a role very symmetrical to the impact niche. It is clear that, in our representation, the two components of the niches have equal role in determining both the existence and the stability of the population dynamical fixed point.

\subsection{Outlook}

Note that the main question of this paper, i.e., that "What is the probability of the possibility of coexistence of a predefined set of species?" is not identical to the empirically more relevant question of "What is the probability distribution of similarity of coexisting species in nature?". The answer to the second question is affected by the distribution of the available species as well as the assembly and evolutionary processes, (e.g. Leibold 1998) which are outside of our discussion.

Nevertheless, we may formulate general expectations based on the presented results. We expect divergence in species specific traits involved in the physiological, behavioral mechanisms determining the impact and sensitivity niches of coexisting species, i.e., in their relations towards the regulating factors. Studies on character displacement (Schluter 2000a; Schluter 2000b, p. 296) demonstrates this empirically in a very convincing way. On the other hand, coexistence is obviously facilitated by similar requirements with respect to the external parameters, i.e., in traits not related to population regulation. (We thank Mathew Leibold for pointing to this dichotomy.) When similarity measures are based on phylogenetic relationships (like in Webb, 2000), these two kinds of similarity are mixed, and the results are indeterminate. We think that the mechanistic approach, that makes explicit the way populations are regulated, is needed for community studies to avoid such confusions.

In this paper we concentrated on the connection between niche overlap and competitive exclusion. This theory should be complemented by analyses on different levels.

On the lower level, one has to understand, how the shape and the breath 
of the niche of a species is determined by the physiological/behavioral constraints (i.e., by the trade-offs) and/or by the evolutionary factors. (See also Chesson, 2000b for the role of trade-offs in coexistence.) Schreiber \& Tobiason (2003) provides an example. They investigated generalist/specialist evolution in the context of the 2-resource Tilman model. Tilman's distinction between essential and substitutable resources - which is related the convex-or-concave nature of the appropriate trade-off (cf. Levins, 1962) but played no role in our discussions because of the linearization - is essential in this analysis. A behavioral constraint (i.e., the fixed level of mobility) is important in determining the spatial niche-breath along an environmental gradient in Mizera \& Meszéna (2003), Mizera et al. (in prep.).

On the higher level, connection to the wider problem of biodiversity is less clear. As an ecosystem is not necessarily saturated, the actual diversity may be determined by the balance between colonization and extinction, as suggested by MacArthur \& Wilson (1967). According to Hubbell (2001), the relation between the niche-assembly and dispersal-assembly perspectives is "... one of the most fundamental unsolved problems in ecology today". Here we attempted to clarify the first one of these two pictures, which is a pre-requisite for reconciliation.

\section{Acknowledgements}

We thanks Sándor Bartha, Niels Bouton, Tamás Czárán, Yoh Iwasa, Patsy Haccou, Éva Kisdi, Kolos Köszeghy, Michel Loreau, István Scheuring, Akira Sasaki, Beáta Oborny and the late Pál Juhász-Nagy for discussions. Comments by Peter Chesson, Mathew Leibold and an anonymous referee on the first version of the ms were helpful. This work was financed form the grants OTKA T033097, T049689 and NWO-OTKA 048.011.039.

\section{References}

Abrams, P. A., 1983. The theory of limiting similarity. Annual Review of Ecology and Systematics 14, 359-376.

Abrams, P. A., 1988. How should resources be counted? Theoretical Population Biology 33, 226-242.

Adler, F. R., 1990. Coexistence of two types on a single resource in discrete time. Journal of Mathematical Biology 28, 695-713.

Anton, H., 1984. Elementary Linear Algebra. John Wiley \& Sons, New York. Apostol, T. M., 1962. Calculus, Volume 2: Calculus of several variables with 
applications to probability and vector analysis. Blaisdell Mathematics Series. Blaisdell Publishing Company.

Armstrong, R. A., McGehee, R., 1976. Coexistence of two competitors on one resource. Journal of Theoretical Biology 56, 499-502.

Armstrong, R. A., McGehee, R., 1980. Competitive exclusion. American Naturalist $115,151-170$.

Begon, M., Harper, J. L., Townsend, C. R., 1996. Ecology. $3^{\text {rd }}$ ed. Blackwell Science Publisher, London.

Bouton, N., Seehausen, O., van Alphen, J. J. M., 1997. Resource partitioning among rock-dwelling haplochromies (Pisces: Cichlidae) from Lake Victoria. Ecology of Freshwater Fish 6, 225-240.

Case, T. J., 2000. An Illustrated Guide to Theoretical Ecology. Oxford University Press.

Caswell, H., 2000. Matrix population methods: Construction, analysis and interpretation. Sinauer Associates.

Chase, J. M., Leibold, M. A., 2003. Ecological niches: linking classical and contemporary approaches. The University of Chicago Press, Chicago.

Chesson, P., 1991. A need for niches? Trends in Ecology and Evolution 6, 26-28.

Chesson, P., 1994. Multispecies competition in variable environment. Theoretical Population Biology 45, 227-276.

Chesson, P., 2000a. General theory of competitive coexistence in spatiallyvarying environments. Theoretical Population Biology 58, 211-237.

Chesson, P., 2000b. Mechanism and maintenance of species diversity. Annual Review of Ecological Systems 31, 343-366.

Christiansen, F. B., 1988. Frequency dependence and competition. Proceedings of the Royal Society of London B 319, 587-600.

Christiansen, F. B., Fenchel, T. M., 1977. Theories of populations in biological communities. Springer-Verlag, Berlin, 1977.

Czárán, T., 1989. Coexistence of competing popualtions along an environmental gradient: a simulation study. Coenoses 4, 113-120.

Czárán, T., 1998. Spatiotemporal models of population and community dynamics. Population and Community Biology series. Chapman \& Hall, 2-6 Boundary Row, London SE1 8HN.

Czárán, T., Bartha, S., 1989. The effect of spatial pattern on community dynamics: a comparison of simulated and field data. Vegetatio 83, 229-39.

Czárán, T., Bartha, S., 1992. Spatio-temporal dynamical models of plant populations and communities. Trends in Ecology and Evolution 7, 38-42.

den Boer, P. J., 1986. The present status of the competitive exclusion principle. Trends in Ecology and Evolution 1, 25-28.

Dieckmann, U., Doebeli, M., 1999. On the origin of species by sympatric speciation. Nature 400, 354-357.

Dieckmann, U., Law, R., 1996. The dynamical theory of coevolution: A derivation from stochastic ecological processes. Journal of Mathematical Biology $34,579-612$. 
Diekmann, O., Gyllenberg, M., Huang, H., Kirkilionis, M., Metz, J. A. J., Thieme, H. R., 2001. On the formulation and analysis of general deterministic structured population models: II. Nonlinear theory. Journal of Mathematical Biology 43, 157-189.

Diekmann, O., Gyllenberg, M., Metz, J. A. J., 2003. Steady state analysis of structured population models. Theoretical Population Biology 63, 309-338.

Diekmann, O., Gyllenberg, M., Metz, J. A. J., Thieme, H. R., 1998. On the formulation and analysis of general deterministic structured population models: I. Linear theory. Journal of Mathematical Biology 36, 349-388.

Doebeli, M., Dieckmann, U., 2003. Speciation along environmental gradient. Nature 421, 259-264.

Edvards, Jr, C. H., 1973. Advanced calculus of several variables. Academic Press, New York London.

Ferrière, R., Gatto, M., 1995. Lyapunov exponents and the mathematics of invasion in oscillatory or chaotic populations. Theoretical Population Biology 48, 126-171.

Galis, F., Metz, J. A., 1998. Why are there so many cichlid species? Trends in Ecology and Evolution 13, 1-2.

Gause, G. F., 1934. The struggle for existence. Williams and Wilkins, Baltimore.

Geritz, S., Kisdi, E., Meszéna, G., Metz, J. A., 2004. Adaptive dynamics of speciation. In: Dieckmann, U., Doebeli, M., Metz, J. A. J., Tautz, D. (Eds.), Adaptive Speciation. Cambridge University Press, Cambridge.

Geritz, S. A., Kisdi, E., 2000. Adaptive dynamics in diploid, sexual populations and the evolution of reproductive isolation. Proceedings of the Royal Society of London B 267, 1671-1678.

Geritz, S. A. H., 1995. Evolutionary stable seed polymorhism and small scale spatial variation in seedling density. American Naturalist 146, 685-707.

Geritz, S. A. H., Kisdi, É., Meszéna, G., Metz, J. A. J., 1998. Evolutionary singular strategies and the adaptive growth and branching of evolutionary trees. Evolutionary Ecology 12, 35-57.

Geritz, S. A. H., Metz, J. A. J., Kisdi, É., Meszéna, G., 1997. The dynamics of adaptation and evolutionary branching. Physical Review Letters 78(10), 2024-2027.

URL http://angel .elte.hu/ geza/GeritzPRL.pdf

Geritz, S. A. H., van der Meijden, E., Metz, J. A. J., 1999. Evolutionary dynamics of seed size and seedling competitive ability. Theoretical Population Biology 55, 324-343.

Goldschmidt, T., Witte, F., de Visser, J., 1990. Ecological segregation in zooplanktivorous haplochromine species (Pisces: Cichlidae) from Lake Victoria. Oikos 58, 343-355.

Gorskov, V. G., Gorshkov, V. V., Makarieva, A. M., 2000. Biotic Regulation of the environment. Key issue of global change. Springer, London.

Greiner, J., Heesterbeek, J. A. P., Metz, J. A. J., 1994. A singular perturbation theorem for evolution equations and time-scale arguments for structured 
population models. Canadian Applied Mathematics Quarterly 2, 435-459.

Gyllenberg, M., Meszéna, G., 2005. On the impossibility of coexistence of infinitely many strategies. Journal of Mathematical Biology 50, 133-160.

Haccou, P., Iwasa, Y., 1995. Optimal mixed strategies in stochastic environments. Theoretical Population Biology 47, 212-243.

Haccou, P., Iwasa, Y., 1998. Robustness of optimal mixed strategies. Journal of Mathematical Biology 36, 485-496.

Hanski, I., 1999. Metapopulation ecology. Oxford series in ecology and evolution. Oxford University Press, Oxford.

Heino, M., Metz, J. A. J., Kaitala, V., 1997. Evolution of mixed maturation strategies in semelparous life-histories: the crucial role of dimensionality of feedback environment. Philosiphical Transactions of the Royal Society of London B, Biological Sciences 353, 1647-1655.

Heino, M., Metz, J. A. J., Kaitala, V., 1998. The enigma of frequencydependent selection. Trends in Ecology and Evolution 13, 367-370.

Hubbel, S. P., 2001. The unified neutral theory of biodiversity and biogeography. Monographs in population biology. Princeton University Press, Princeton.

Huisman, J., Weissing, F. J., 1999. Biodiversity of plancton by species oscillation and chaos. Nature 402, 407-410.

Huston, M. A., 1979. A general hypothesis of species diversity. American Naturalist 113, 81-101.

Huston, M. A., 1994. Biological Diversity. The coexistence of species on changing landscapes. Cambridge University Press, Cambridge.

Hutchinson, G. E., 1959. Homage to Santa Rosalia, or why are so many kinds of animals? American Naturalist XCIII (870), 137-145.

Hutchinson, G. E., 1961. The paradox of the plankton. American Naturalist XCV (882), 137-145.

Hutchinson, G. E., 1978. An introduction to population ecology. Yale University Press, New Haven and London.

Károlyi, G., Péntek, Á., Scheuring, I., Tél, T., Toroczkai, Z., 2000. Chaotic flow: the physics of species coexistence. Proceedings of the National Academy of Sciences USA 97, 13661-13665.

Károlyi, G., Scheuring, I., Czárán, T., 2002. Metabolic networks dynamics in open chaotic flow. Chaos 12, 460-469.

Kisdi, E., Geritz, S. A., 1999. Adaptive dynamics in allele space: evolution of genetic polymorphism by small mutations in a heterogeneous environment. Evolution 53, 993-1008.

Kisdi, É., Meszéna, G., 1993. Density dependent life history evolution in fluctuating environment. In: Yoshimura, J., Clark, C. W. (Eds.), Adaptation in Stochastic Environments. Vol. 98 of Lecture Notes in Biomathematics. Springer-Verlag, pp. 26-62.

Kitzing, A. P., Levin, S. A., Dushof, J., Pacala, S., 1999. Limiting similarity, species packing system stability for hierarchical competition-colonization models. American Naturalist 153, 371-383. 
Köszeghy, K., 2004. An operative formalization of the concept of ecological niche. Master thesis, in Hungarian.

Krebs, C. J., 2001. Ecology. The experimental analysis of distribution and abundance. Benjamin Cummings, San Francisco, California.

Leibold, M. A., 1995. The niche concept revisited: mechanistic models and community context. Ecology 76 (5), 1371-1382.

Leibold, M. A., 1998. Similarity and local co-existence of species in regional biotas. Evolutionary Ecology 12, 95-110.

Levin, S. M., 1970. Community equlibria and stability, and an extension of the competitive exclusion principle. American Naturalist 104(939), 413-423.

Levins, R., 1962. Theory of fitness in a heterogeneous environment i. the fitness set and adaptive function. American Naturalist 96, 361-373.

Levins, R., 1979. Coexistence in a variable environment. American Naturalist 114, 765-783.

Loreau, M., 1989. Coexistence of temporally segregated competitors in a cyclic environment. Theoretical Population Biology 36(2), 181-201.

Loreau, M., 1992. Time scale of resource dynamics and coexistence through time partitioning. Theoretical Population Biology 41(3), 401-412.

Loreau, M., Ebenhöh, W., 1994. Comptetitive exclusion and coexistence of species with complex life cycles. Theoretical Population Biology 46(1), 5877.

MacArthur, R. H., 1958. Population ecology of some warblers of northeastern coniferous forests. Ecology 39, 599-619.

MacArthur, R. H., 1962. Some generalized theorems of natural selection. Proceedings of the National Academy of Sciences USA 48, 1893-97.

MacArthur, R. H., Levins, R., 1964. Competition, habitat selection and character displacement in a patchy environment. Proceedings of the National Academy of Sciences USA 51, 1207-1210.

MacArthur, R. H., Levins, R., 1967. The limiting similarity, convergence, and divergence of coexisting species. American Naturalist 101 (921), 377-385.

May, R. M., 1973. Stability and Complexity in Model Ecosystems. Princeton University Press, Princeton.

May, R. M., 1974. On the theory of niche overlap. Theoretical Population Biology 5, 297-332.

May, R. M., MacArthur, R. H., 1972. Niche overlap as a function of environmental variability. Proceedings of the National Academy of Sciences USA 69, 1109-1113.

Maynard Smidth, J., Szathmáry, E., 1995. The major transitions in evolution. W.H. Freeman Spektrum, Oxford.

Meszéna, G., Czibula, I., Geritz, S. A. H., 1997. Adaptive dynamics in a 2patch environment: a toy model for allopatric and parapatric speciation. Journal of Biological Systems 5, 265-284.

URL http: //angel.elte.hu/ geza/MeszenaEtal1997.pdf

Meszéna, G., Gyllenberg, M., Jacobs, F. J., Metz, J. A. J., in press. Link between population dynamics and dynamics of darwinian evolution. Physical 
Review Letters.

URL http://angel .elte.hu/ geza/PRLMeszena2005.pdf

Meszéna, G., Metz, J. A. J., 1999. Species diversity and population regulation: the importance of environmental feedback dimensionality. IIASA Working Paper WP-99-045.

URL http://www.iiasa.ac.at/cgi-bin/pubsrch?IR99045

Meszéna, G., Szathmáry, E., 2001. Adaptive dynamics of parabolic replicators. Selection 2, 147-159.

URL http://angel.elte.hu/ geza/FEJ-10.PDF

Metz, J. A. J., Diekmann, O., 1986. The dynamics of physiologically structured populations. Vol. 68 of Lecture Notes in Biomathematics. Springer, Berlin.

Metz, J. A. J., Geritz, S. A. H., Meszéna, G., Jacobs, F. J. A., van Heerwaarden, J. S., 1996a. Adaptive dynamics, a geometrical study of the consequences of nearly faithful reproduction. In: van Strien, S. J., Verduyn Lunel, S. M. (Eds.), Stochastic and spatial structures of dynamical systems. North Holland, pp. 183-231.

Metz, J. A. J., Geritz, S. A. H., Nisbet, R. M., 1992. How should we define "fitness" for general ecological scenarios? Trends in Ecology and Evolution 7, 198-202.

Metz, J. A. J., Mylius, S. D., Diekmann, O., 1996b. When does evolution optimise? On the relation between types of density dependence and evolutionarily stable life history parameters. IIASA Working Paper WP-96-004. URL http://www.iiasa.ac.at/cgi-bin/pubsrch?WP96004

Misher, C. W., Thorne, K. S., Wheeler, A., 1973. Gravitation. H. W. Freeman and Company, New York.

Mizera, F., Meszéna, G., 2003. Spatial niche packing, character displacement and adaptive speciation along an environmental gradient. Evolutionary Ecology Research 5, 363-382.

Mizera, F., Meszéna, G., Czárán, T., Iwasa, Y., in prep. Niche-segregation and transition zone along an environmental gradient.

Parvinen, K., Meszéna, G., in prep. Disturbance-generated niche-segregation in a structurred metapopulation model.

Petraitis, P. S., 1989. The representation of niche breadth and overlap on Tilman's consumer-resource graphs. Oikos 56, 289-292.

Rado, T., Reichelderfer, P. V., 1995. Continuous transformations in analysis with an introduction to algebraic topology. Springer-Verlag, Berlin.

Rosenzweig, M. L., 1995. Species diversity in space and time. Cambridge University Press, Cambridge.

Roughgarden, J., 1979. Theory of population genetics and evolutionary ecology. Macmillan, New York.

Rudin, W., 1974. Real and complex analysis. Second edition. McGraw-Hill.

Sasaki, A., 1997. Clumped distribution by neighborhood competition. Journal of Theoretical Biology 186, 415-430.

Sasaki, A., Ellner, S., 1995. The evolutionarily stable phenotype distribution in a random environment. Evolution 49(2), 337-350. 
Scheuring, I., Czárán, T., Szabó, P., Károlyi, G., Toroczkai, Z., 2003. Spatial models of prebiotic evolution: soup before pizza? Origins of Life and Evolution of Biosphere 33, 319-355.

Schluter, D., 2000a. Ecological character displacement in adaptive radiation. American Naturalist 156, S4-S16.

Schluter, D., 2000b. The Ecology of Adaptive Radiation. Oxford University Press, Oxford.

Schreiber, S. J., Tobiason, G. A., 2003. The evolution of resource use. Journal of Mathematical Biology 47, 56-78.

Seehausen, O., Bouton, N., 1997. Microdistribution and fluctuations in niche overlap in a rocky shore cichlid community in Lake Victoria. Ecology of Freshwater Fish 6, 161-173.

Stromberg, K. R., 1981. An introduction to classical real analysis. Wadsworth International Group, Belmont, California.

Szabó, P., Meszéna, G., submitted. Limiting similarity revisited.

Szabó, P., Scheuring, I., Czárán, T., Szathmáry, E., 2002. In silico simulations reveal that replicators with limited dispersal evolve towards higher efficiency and fidelity. Nature 420, 360-363.

Tél, T., Károlyi, G., Péntek, Á., Scheuring, I., Toroczkai, Z., Grebogi, C., Kadtke, J., 2000. Chaotic advection, diffusion, and reactions in open flows. Chaos 10(1), 89-98.

Tilman, D., 1982. Resource Equilibrium and Community Structure. Princeton University Press, Princeton.

Tilman, D., Mattson, M., Langer, S., 1981. Competition and nutrient kinetics along a temperature gradient: an experimental test of a mechanistic approach to niche theory. Limnology and Oceanography 26, 1020-1033.

Tilman, D., May, R. M., Lehman, C. L., Nowak, M., 1994. Habitat destruction and the extinction debth. Nature 371, 65-66.

Tuljapurkar, S., 1990. Population dynamics in variable environments. Vol. 85 of Lecture Notes in Biomathematics. Springer-Verlag, Berlin.

Turchin, P., 1999. Population regulation: a synthetic view. Oikos 84, 153-159.

Turchin, P., 2003. Complex population Dynamics. Princeton University Press.

Turner, G. F., 1999. What is a fish species? Reviews in Fish Biology and Fisheries 9, 281-297.

Vandermeer, J. H., 1975. Interspecific competition: A new approach to the classical theory. Science 188, 253-255.

Vukics, A., Asbóth, J., Meszéna, G., 2003. Speciation in multidimensional evolutionary space. Physical Review E 68, 041903.

URL http://angel .elte.hu/ geza/PRE41903.pdf

Webb, C. O., 2000. Exploring the phylogenetic structure of ecological communities: An example for rain forest trees. American Naturalist 156, 145-155.

Yodzis, P., 1989. Introduction to theoretical ecology. Harper \& Row, New York. 


\section{Appendix}

Section A introduces the basic notions in more precise terms. Section B attempts to de-mystify the concept of tensors in this context, which will be needed to calculate the volume of the parellelepiped in Section C.

\section{A Unifying the finite and infite dimensional cases: the basic no- tions}

Let $\Omega$ denote the set of the (descriptors of the) regulating factors. $D$ is the number of these factors. When $D=\infty, \Omega$ is usually a subset of $\mathbb{R}^{n}$. The choice $\Omega=\mathbb{R}$ corresponds to the one-dimensional resource continuum of Section 3.4.

The state of the regulating environment, denoted by $\boldsymbol{I}$, is specified when the value of each regulating variable is given. Consequently, $\boldsymbol{I}$ can be considered as a mapping from $\Omega$ to $\mathbb{R}$. By using the usual notation $\mathbb{R}^{\Omega}$ for the set of all functions from $\Omega$ to $\mathbb{R}$,

$$
\boldsymbol{I} \in \mathbb{R}^{\Omega}: \quad x \in \Omega \mapsto I(x) \in \mathbb{R} .
$$

When $D$ is finite, $\Omega$ is identified with the index set $\{1,2, \ldots, D\}$ and $\mathbb{R}^{\Omega}$ becomes $\mathbb{R}^{D}$. In this case, $\boldsymbol{I}$ corresponds to a finite dimensional vector:

$$
\boldsymbol{I}=\left(I_{1}, I_{2}, \ldots, I_{D}\right) \in \mathbb{R}^{D} .
$$

Analogously, the impact and the sensitivity vectors/functions $\boldsymbol{C}_{j}$ and $\boldsymbol{S}_{j}$ for the population $j$ are elements of $\mathbb{R}^{\Omega}$, or $\mathbb{R}^{D}$ when $D$ is finite. Both of them assign a value to each regulating factor: the $j$ th population's impact on, or the sensitivity towards, the specific factor.

The scalar product for these vectors/functions in $\mathbb{R}^{\Omega}$, measuring their overlap, is defined in the usual way:

$$
\boldsymbol{u} \cdot \boldsymbol{v}= \begin{cases}\sum_{i=1}^{D} u_{i} v_{i} & \Omega \text { is finite } \\ \int u(x) v(x) d x & \Omega=\mathbb{R}^{n}\end{cases}
$$

The absolute value, or norm, of a vector/function is

$$
|u|=\sqrt{u \cdot u} .
$$

A mathematical caveat is needed here though: For the infinite dimensional 
case, the existence of the "overlap" integral in (A.3) is an additional requirement, which restricts the allowed functions $\boldsymbol{I}$ to the so-called $L_{2}$ space.

In some applications, the set $\Omega$ can be more complicated than $\mathbb{R}^{n}$. Always, a prescription for the scalar product is needed, which makes the allowable subset of $\mathbb{R}^{\Omega}$ a Hilbert space (Rudin, 1974, p. 76). For $D=\infty$, the definition (43) of the sensitivity vector/function and the chain rule (47) relies on the Riesz representation theorem for Hilbert spaces (Theorem 4.12. in Rudin, 1974, p. $85)$.

\section{B Tensors light}

While tensor calculus is usually considered hard, the parts of it that we need are very simple. From our point of view, an $L$-tensor is a function with $L$ arguments in $\Omega$.

The tensor product, denoted by $\circ$, of $L$ functions $\boldsymbol{u}_{1}, \boldsymbol{u}_{2}, \ldots, \boldsymbol{u}_{L} \in \mathbb{R}^{\Omega}$ is an $L$ tensor:

$$
\left(\boldsymbol{u}_{1} \circ \boldsymbol{u}_{2} \circ \ldots \circ \boldsymbol{u}_{L}\right)\left(x_{1}, x_{2}, \ldots, x_{L}\right)=u_{1}\left(x_{1}\right) u_{2}\left(x_{2}\right) \ldots u_{L}\left(x_{L}\right)
$$

When $\Omega$ is finite, the tensor product is a quantity with $L$ indices, i.e., an " $L$ dimensional matrix":

$$
\left(\boldsymbol{u}_{1} \circ \boldsymbol{u}_{2} \circ \ldots \circ \boldsymbol{u}_{L}\right)_{j_{1}, j_{2}, \ldots, j_{L}}=u_{1 j_{1}} u_{2 j_{2}} \ldots u_{L j_{L}}
$$

where $u_{l j}$ denotes the $j$ th component of the vector $\boldsymbol{u}_{l}$. It looks frightening, but no deep issues are involved here. Note that tensor product is identical to the usual dyadic product of matrix calculus when $L=2$. In this case, the result is a two-dimensional matrix.

The scalar product and norm of tensors is defined analogously to Eqs. (A.3) and (A.4) by extending the integration/summation to all variables/indices. In particular, the scalar product of two tensor products can be evaluated componentwise, as

$$
\begin{aligned}
& \left(\boldsymbol{u}_{1} \circ \boldsymbol{u}_{2} \circ \ldots \circ \boldsymbol{u}_{L}\right) \cdot\left(\boldsymbol{v}_{1} \circ \boldsymbol{v}_{2} \circ \ldots \circ \boldsymbol{v}_{L}\right)= \\
& \quad=\left(\boldsymbol{u}_{1} \cdot \boldsymbol{v}_{1}\right)\left(\boldsymbol{u}_{2} \cdot \boldsymbol{v}_{2}\right) \ldots\left(\boldsymbol{u}_{L} \cdot \boldsymbol{v}_{L}\right) .
\end{aligned}
$$




\section{Volume of parallelepiped}

The (L-dimensional) volume $\mathcal{V}_{\boldsymbol{u}}$ of the parallelepiped, spanned by the $L$ vectors $\boldsymbol{u}_{1}, \boldsymbol{u}_{2}, \ldots, \boldsymbol{u}_{L}$, has a central role in our analysis. The issue is usually discussed in the context of differential geometry and general relativity (see, for instance, Edwards, Jr., 1973, p. 345; Misher et al., 1973, p. 204). For the convenience of the reader, we summarize the necessary mathematics here.

The case $L=D$ is well-known (Apostol, 1962, pp. 84-86; Case, 2000, pp. 420-422; Edwards, Jr., 1973, p. 245). The volume can be calculated as the determinant of the square matrix $\boldsymbol{u}$ formed from the vectors, i.e.,

$$
\mathcal{V}_{\boldsymbol{u}}=\operatorname{det} \boldsymbol{u}=\sum_{\sigma \in \Sigma_{L}}(-1)^{\sigma} u_{1 \sigma(1)} \cdot u_{2 \sigma(2)} \cdot \ldots \cdot u_{L \sigma(L)} \quad \text { for } \quad L=D
$$

Here, the generic element $u_{i j}$ of the matrix $\boldsymbol{u}$ is the $j$ th component of the $i$ th vector $\boldsymbol{u}_{i}$. In the expansion of the determinant, $\Sigma_{L}$ denotes the group of the permutations of the index set $\{1, \ldots, L\}$ and $(-1)^{\sigma}$ is \pm 1 if the permutation $\sigma$ is even or odd. We have to generalize this formula to $L \neq D$, when the matrix $\boldsymbol{u}$ is not a square matrix and the determinant has no sense. ( $D$ is allowed to be infinite, $L$ is not.)

We will show that the volume of the parallelepiped spanned by $L$ vectors $\boldsymbol{u}_{1}, \boldsymbol{u}_{2}, \ldots, \boldsymbol{u}_{L}$ can be written as

$$
\mathcal{V}_{\boldsymbol{u}}=\left|\boldsymbol{u}_{1} \wedge \boldsymbol{u}_{2} \wedge \ldots \wedge \boldsymbol{u}_{L}\right|
$$

i.e., it is the norm of the so-called wedge product of the vectors. Wedge product is defined as the $L$-tensor

$$
\boldsymbol{u}_{1} \wedge \boldsymbol{u}_{2} \wedge \ldots \wedge \boldsymbol{u}_{L}=\frac{1}{\sqrt{L !}} \sum_{\sigma \in S^{L}}(-1)^{\sigma} \boldsymbol{u}_{\sigma(1)} \circ \boldsymbol{u}_{\sigma(2)} \circ \ldots \circ \boldsymbol{u}_{\sigma(L)} .
$$

That is, $\wedge$ can be interpreted as the unique anti-symmetrized tensor product of the vectors. The prefactor $1 / \sqrt{L}$ ! is chosen for our later convenience. Observe the similarity to the definition of the determinant.

To develop some intuition for the wedge product first consider a special case. For $L=2$, the wedge product can be written as

$$
\boldsymbol{u} \wedge \boldsymbol{v}=\frac{1}{\sqrt{2}}(\boldsymbol{u} \circ \boldsymbol{v}-\boldsymbol{v} \circ \boldsymbol{u})
$$

or, for finite $D$, as

$$
(\boldsymbol{u} \wedge \boldsymbol{v})_{i j}=\frac{1}{\sqrt{2}}\left(u_{i} v_{j}-u_{j} v_{i}\right)
$$


which is an $L \times L$ antisymmetric matrix. In the special case of $D=3$, the antisymmetric matrix (C.5) has 6 non-zero components, which are, after multiplication with $\pm \sqrt{2}$, identical to the 3 components of the vector product $\boldsymbol{u} \times \boldsymbol{v}$. (See Anton, 1984, p. 111 for the vector product.) Then, it is clear that

$$
|\boldsymbol{u} \wedge \boldsymbol{v}|=|\boldsymbol{u} \times \boldsymbol{v}|=|\boldsymbol{u}||\boldsymbol{v}| \sin (\boldsymbol{u}, \boldsymbol{v}) .
$$

In line with our claim, this is just the area of the parallelogram spanned by the vectors $\boldsymbol{u}$ and $\boldsymbol{v}$ [c.f. Eq. (22)]. The wedge product can be seen as a generalization of the vectorial product, which itself is a peculiarity of the 3 dimensional spaces.

When the $D$ is finite, one can spell the general wedge product (C.3) out in index notation, as

$$
\begin{aligned}
& \left(\boldsymbol{u}_{1} \wedge \boldsymbol{u}_{2} \wedge \ldots \wedge \boldsymbol{u}_{L}\right)_{j_{1}, j_{2}, \ldots, j_{L}}= \\
& \quad=\frac{1}{\sqrt{L !}} \sum_{\sigma \in S^{L}}(-1)^{\sigma} u_{\sigma(1) j_{1}} \cdot u_{\sigma(2) j_{2}} \cdot \ldots \cdot u_{\sigma(L) j_{L}} .
\end{aligned}
$$

In this $L$-tensor, the only elements that can be non-zero are those where the indices $j_{1}, j_{2}, \ldots, j_{L}$ are all different. To prove (C.2), we discuss three cases.

For $D<L$, the wedge product vanishes, as there are no $L$ different indices. This is in line with the fact that the $L$ dimensional volume $\mathcal{V}_{\boldsymbol{u}}$ is zero in $D<L$ dimensions.

For $D=L$, each non-zero element equals to $\pm \operatorname{det} \boldsymbol{u} / \sqrt{L !}$, where the square matrix $\boldsymbol{u}$ is built from the vectors $\boldsymbol{u}_{i}$. As we have $L$ ! of them, the norm of the wedge product is

$$
\left|\boldsymbol{u}_{1} \wedge \boldsymbol{u}_{2} \wedge \ldots \wedge \boldsymbol{u}_{L}\right|=|\operatorname{det} \boldsymbol{u}|=\mathcal{V}_{\boldsymbol{u}} \quad \text { for } L=D
$$

For $D>L, \boldsymbol{u}$ is no longer a square matrix and $\operatorname{det} \boldsymbol{u}$ has no meaning. Nevertheless, the expression (C.2) remains valid, as one can apply the argument, leading to Eq. (C.8), in the $L$ dimensional subspace of $\mathbb{R}^{\Omega}$ containing the vectors $\boldsymbol{u}_{i}$. This proves our claim. 
The Proposition of Section 3.3 is a direct consequence of the relation

$$
\begin{aligned}
& \left(\boldsymbol{S}_{1} \wedge \boldsymbol{S}_{2} \wedge \ldots \boldsymbol{S}_{L}\right) \cdot\left(\boldsymbol{C}_{1} \wedge \boldsymbol{C}_{1} \wedge \ldots \wedge \boldsymbol{C}_{L}\right)= \\
= & \left(\frac{1}{\sqrt{L !}} \sum_{\sigma \in S^{L}}(-1)^{\sigma} \boldsymbol{S}_{\sigma(1)} \circ \ldots \circ \boldsymbol{S}_{\sigma(L)}\right)\left(\frac{1}{\sqrt{L !}} \sum_{\sigma^{\prime} \in S^{L}}(-1)^{\sigma^{\prime}} \boldsymbol{C}_{\sigma^{\prime}(1)} \circ \ldots \circ \boldsymbol{C}_{\sigma^{\prime}(L)}\right)= \\
= & \frac{1}{L !} \sum_{\sigma \in S^{L}} \sum_{\sigma^{\prime} \in S^{L}}(-1)^{\sigma \sigma^{\prime}}\left(\boldsymbol{S}_{\sigma(1)} \cdot \boldsymbol{C}_{\sigma^{\prime}(1)}\right)\left(\boldsymbol{S}_{\sigma(2)} \cdot \boldsymbol{C}_{\sigma^{\prime}(2)}\right) \ldots\left(\boldsymbol{S}_{\sigma(L)} \cdot \boldsymbol{C}_{\sigma^{\prime}(L)}\right)= \\
\quad= & \sum_{\sigma \in S^{L}}(-1)^{\sigma}\left(\boldsymbol{S}_{1} \cdot \boldsymbol{C}_{\sigma(1)}\right)\left(\boldsymbol{S}_{2} \cdot \boldsymbol{C}_{\sigma(2)}\right) \ldots\left(\boldsymbol{S}_{L} \cdot \boldsymbol{C}_{\sigma(L)}\right)=\operatorname{det} \boldsymbol{a}, \quad(\mathrm{C} .9)
\end{aligned}
$$

where the matrix $\boldsymbol{a}$ is defined by

$$
a_{i j}=\boldsymbol{S}_{i} \cdot \boldsymbol{C}_{j}
$$

in line with Eqs. (12) and (47).

Finally, we must demonstrate that

$$
(\boldsymbol{u} \wedge \boldsymbol{v})^{2}+(\boldsymbol{u} \cdot \boldsymbol{v})^{2}=\boldsymbol{u}^{2} \boldsymbol{v}^{2}
$$

which is the basis of Eq. (25). Consider, that

$$
(\boldsymbol{u} \wedge \boldsymbol{v})^{2}=\frac{1}{2}(\boldsymbol{u} \circ \boldsymbol{v}-\boldsymbol{v} \circ \boldsymbol{u})^{2}=(\boldsymbol{u} \circ \boldsymbol{v})^{2}-[(\boldsymbol{u} \circ \boldsymbol{v})(\boldsymbol{v} \circ \boldsymbol{u})]
$$

Then, the equalities

$$
(\boldsymbol{u} \circ \boldsymbol{v})^{2}=\boldsymbol{u}^{2} \boldsymbol{v}^{2}
$$

and

$$
(\boldsymbol{u} \circ \boldsymbol{v})(\boldsymbol{v} \circ \boldsymbol{u})=(\boldsymbol{u} \cdot \boldsymbol{v})^{2}
$$

prove the statement.

As an illustration we evaluate the area of the parallelogram for the resource continuum case of Section 3.4, i.e., for $\Omega=\mathbb{R}, L=2$ :

$$
\mathcal{V}_{\boldsymbol{u}}=\left|\boldsymbol{u}_{1} \wedge \boldsymbol{u}_{2}\right|=\sqrt{\int_{x_{2}} \int_{x_{1}}\left[\frac{u_{1}\left(x_{1}\right) u_{2}\left(x_{2}\right)-u_{1}\left(x_{2}\right) u_{2}\left(x_{1}\right)}{\sqrt{2}}\right]^{2} d x_{1} d x_{2} .}
$$

Straightforward calculation connects this expression to the overlap integral:

$$
\mathcal{V}_{\boldsymbol{u}}=\sqrt{\int_{x}\left[u_{1}(x)\right]^{2} d x \cdot \int_{x}\left[u_{2}(x)\right]^{2} d x-\left[\int_{x} u_{1}(x) u_{2}(x) d x\right]^{2}} .
$$

This is in line with the formulas (25) and (C.11): the area decreases with increasing overlap. 